
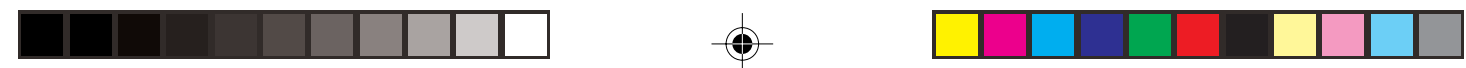

\title{
LISTA DE ESPECIES, ENDEMISMO Y CONSERVACIÓN DE LOS DE MAMÍFEROS DE COSTA RICA
}

\author{
BERNAL RODRÍGUEZ-H ${ }^{1}$, FEDERICO A. CHINCHILLA ${ }^{2}$ \\ Y LAURA J. MAY-COLLADO ${ }^{3}$
}

${ }^{1}$ Museo Nacional de Costa Rica, 749-1000 San José, Costa Rica, bernalr@racsa.co.cr.

${ }^{2}$ Instituto de Ecología, Universidad Autónoma de México. Dirección actual: Estación

Biológica Monteverde, Apo. 22 -5655, Monteverde, Puntarenas Costa Rica

${ }^{3}$ Department of Biological Sciences, Florida International University UP Miami, FL 33199

\begin{abstract}
The geographical position of Costa Rica has historically played an important role in the composition and diversity of our mammalian fauna, consisting of species originating both in the Neartic and Neotropical regions. Based on published data, new descriptions and reports, we here present an updated list of all terrestrial and aquatic Costa Rican mammals. We summarize available information on their status, and present our concerns regarding those species with populations under alarming conditions. The mammalian fauna of Costa Rica is mainly Neotropical. A total of 238 species, grouped in 140 genera and 44 families, were documented. The order Chiroptera is the most diverse, followed by Rodentia, Cetacea, and Carnivora. About $7 \%$ of the species are endemic to Costa Rica (or Costa Rica-Nicaragua, Costa Rica-Panamá). Unfortunately, despite of all the efforts to protect our fauna and flora, threat to Costa Rican mammals is increasing, and a number of species have gone, and are going extinct. As in most other areas, habitat loss, both in terrestrial and coastal ecosystems, poses the greatest threat to mammalian diversity of Costa Rica. Other factors, such as poaching, inappropriate gear used in fisheries, and other human exploitation are also threatening mammal populations in Costa Rica. Future conservation efforts should focus on more detailed conservation and management strategies, particularly of large mammals (e.g. monkeys, felids).
\end{abstract}

Palabras clave: Costa Rica, riqueza de especies, mamíferos, conservación, endemismo.

\section{INTRODUCCIÓN}

Debido a su posición geográfica, Costa Rica ha servido como puente y filtro para la distribución de especies de mamíferos provenientes tanto de la región Neártica como de la Neotropical. Como resultado, la mastofauna actual del país esta compuesta por una mezcla de especies provenientes del norte y sur de América; por ejemplo, algunos carnívoros como el coyote (Canis latrans) y los ratones de la familia Heteromyidae son de origen Neártico; por el contrario los osos hormigueros, perezosos y monos, entre otros, son animales endémicos para el Neotrópico. 
Como consecuencia del origen mixto de las especies y, en relación con su pequeña extensión geográfica $\left(50,900 \mathrm{~km}^{2}\right)$, se puede decir que Costa Rica presenta una alta diversidad de mamíferos.

Esta diversidad de mamíferos, comienza a documentarse desde mediados del siglo XIX con la formación de las primeras colecciones y listas de especies, en trabajos como los de Frantzius (1869), Alston (1879), Alfaro (1897) y Goodwin (1946). El conocimiento de la riqueza de especies en Costa Rica, continúa siendo activo, y en los últimos años varios autores (Janzen, 1983; Wilson, 1983; AcevedoGutierrez, 1996; Rodríguez y Chinchilla, 1996; Carrillo et al., 2000; Mora, 2000; Rodríguez-Fonseca, 2001; Wilson et al., 2002) han publicado listas de especies de mamíferos, adicionando nueva información para el país. El presente trabajo recopila los últimos cambios en la sistemática y filogenia, descripción de especies nuevas y registros de ocurrencia recientes para el país, tanto de mamíferos terrestres como marinos.

\section{MÉTODOS}

Para elaborar la lista de especies, tomamos como base los trabajos de Wilson (1983), Rodríguez y Chinchilla (1996), Rodríguez-H y Wilson (1999), Wilson et al. (2002), Ceballos et al. (2002), Leatherwood y Reeves (1983), Acevedo-Gutierréz (1996), así como la base de datos de Cetáceos (1975-2001) para Costa Rica del Departamento de Pesquería (Southwest Fisheries Science Service) de los Estados Unidos.

Las categorías de conservación para las especies fueron obtenidas de la Unión Mundial para la Naturaleza, UICN (1999), y de la Lista Roja de la UICN (2000) (http://www.redlist.org/ ).

De manera general, seguimos la nomenclatura propuesta por Wilson y Reeder (1993), con las siguientes cambios y comentarios:

1-Seguimos la reorganización de las familias de Didelphimorphia propuesta por Hershkovitz (1992).

2-Separamos a Cryptotis merriami de Cryptotis nigrescens, de acuerdo a Woodman y Timm (1993) y Woodman (2000).

3-La relaciones filogenéticas dentro de la familia Phyllostomidae han estado en discusión en los recientes años. Diferentes investigaciones con técnicas moleculares y morfométricas obtuvieron diferentes resultados, (vg. McKenna y Bell, 1997; Baker et al., 2000; Wetterer et al., 2000). Nosotros seguimos la clasificación de Wetterer et al. (2000).

4-Seguimos Simmons y Handley (1998) quienes reorganizan el género Centronycteris, correspondiendo a Costa Rica la especie C. centralis. 
5-Consideramos que lo que se conocía como Micronycteris en realidad es más de un género, siguiendo lo propuesto por Simmons y Voss (1998) y Wetterer et al. (2000). 6-Seguimos lo propuesto por Simmons (1996) quien reorganizó las especies de Micronycteris con banda interaucular, correspondiendo a Costa Rica M. microtis y no el nombre anteriormente propuesto M. megalotis.

7-Incluimos la especie Carollia sowelli como especie diferente de C. brevicauda, siguiendo a Baker et al. (2002). La presencia de C. brevicauda en Costa Rica está por confirmarse.

8-De acuerdo a Lee et al. (2002), adoptamos el género Lophostoma para las especies Tonatia brasiliensis y T. silvicola.

9-Seguimos a Handley (1987) y Owen (1987), quienes consideran Artibeus watsoni una especie distinta de Artibeus glaucus.

10-Incluimos Chiroderma trinitatum, registrado para Costa Rica por Timm y LaVal (1998)

11-Reconocemos a Enchisthenes como un género diferente de Artibeus, siguiendo a Arroyo-Cabrales y Owen (1997) y Wetterer et al. (2000).

12-Seguimos a Wetterer et al. (2000) que incluyen a Mesophylla macconnelli como una especie del género Ectophylla.

13-Sturnira ludovici es una especie reconocida en realidad como un complejo de especies que se encuentra en revisión; posiblemente en Costa Rica existan más de una especie de este complejo. Por ahora aceptamos que para Centroamérica, el nombre Sturnira hondurensis propuesto por Goodwin (1940) es lo correcto (ver Iudica, 2000) 14-Utilizamos Simmons (1998) para el uso de la Subfamilia Myotinae y Familia Antrozoidae.

15-Incluímos Lasiurus intermedius registrado por Rodríguez-H et al. (en prensa).

16-Después de revaluar el estado taxonómico y nomenclatural de la especie Molossus bondae, López-González y Presley (2001) proponen que el nombre correcto es Molossus currentium.

17-Utilizamos el cambio propuesto por Peters et al. (2002), que proponen que el nombre válido para el género Molossops en el norte de Latinoamérica es el género Cynomops.

18-Siguiendo a la International Commission on Zoological Nomenclature (ISCN, 1998) reorganizamos la Familia Cuniculidae y el género Cuniculus para la especie Agouti paca.

19-Aceptamos el uso de los nombres de la familia Atelidae y subfamilia Mycetinae según lo propuesto por Groves (2001).

20-Utilizamos el nombre de la familia Mephitidae, siguiendo a Dragoo y Honeycutt (1997).

21-No incluímos en la lista a Bassaricyon lasius, descrita por Harris en 1932, debido a que el holotipo es el único ejemplar que se conoce y se tiene más de 70 años sin 
ningún otro tipo de registro. Creemos que se debe revisar este ejemplar para asegurarse su validez como especie diferente.

22-Mantenemos el nombre de Tayassu tajacu siguiendo a Wright (1989).

\section{RESULTADOS Y DISCUSIÓN}

\section{Composición de especies}

Se han registrado 238 especies de mamíferos, de las cuales 207 son especies terrestres. Los órdenes con mayor número de especies son: Chiroptera con 109 especies (53\% de las especies terrestres), seguido por Rodentia con 45 especies, Cetacea con 29 especies y Carnivora con 23 especies. Esta diversidad se organiza en 140 géneros y 44 familias (Cuadro 1).

Para ilustrar qué tan exclusivas son las especies de mamíferos de Costa Rica, comparamos con la lista publicada por Ceballos et al. (2002) para México; el porcentaje de similitud (Indice de Jaccard x 100) entre ambos países es del 29\%. Esto se debe a la diferencia en tamaños entre ambos países (Costa Rica es similar al estado de Quintana Roo) y por supuesto a la diferente posición geográfica, que le da a México un alto y mayor número de especies neárticas que Costa Rica. Con el mismo ejercicio, comparando a Costa Rica con un país neotropical y de similar tamaño como lo es Guyana (lista de especies de Engstrom y Lim, 2002), se encuentra que la similitud es del $25 \%$. Esto indica que en Costa Rica la mayoría de especies son de origen neotropical pero en su composición de especies, existe una importante proporción de mamíferos que son propios de Mesoamérica, y solo se encuentran desde el sur de México hasta Panamá.

\section{Endemismo}

En Costa Rica dentro de sus límites políticos se encuentran cinco especies de mamíferos endémicos (Orthogeomys heterodus, O. cherrie, O. underwoodi, Heteromys oresterus y Reithrodontomys rodriguezi), pero si se toma en cuenta la región biogeográfica de la Cordillera de Talamanca-Chiriquí (tierras altas al sur de Costa Rica y oeste de Panamá), este número aumenta a 17. También Costa Rica y Nicaragua comparten la pequeña distribución de Reithrodontomys brevirostris, lo cual aumenta a 18 el número de especies endémicas regionales (Cuadro 2).

El endemismo de mamíferos de Costa Rica se presenta principalmente en tierras altas, en los pisos Montano alto, Subalpino y Alpino. De las 18 especies, 16 especies se distribuyen en elevaciones superiores a $1500 \mathrm{msnm}$, encontradas principalmente en la Cordillera de Talamanca. Esta cordillera, compartida entre Costa Rica y Panamá, alcanza su mayor elevación a los 3819 msnm, en el Cerro Chirripó y 


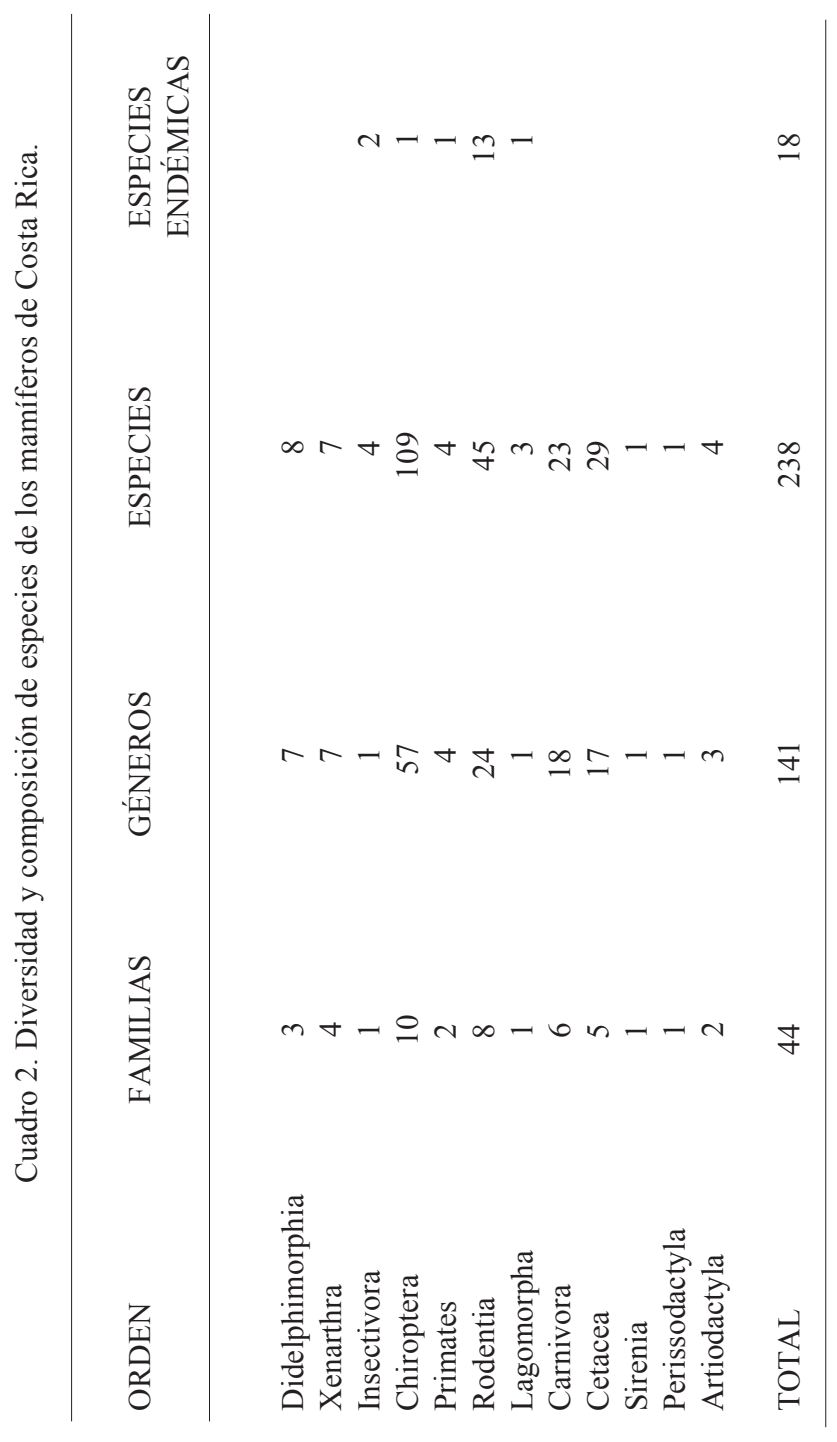


representa el límite norte de los Páramos. Las regiones altas de Talamanca son consideradas altamente diversas en plantas (Whitmore, 1990; Harcout y Sayer, 1996), ya que albergan tal vez más de 10000 especies de plantas vasculares. Tambien es una importante área de endemismo de aves en el Neotrópico (Harcourt y Sayer, 1996). Además de este importante componente de endemismo, Talamanca es la región con la mayor cobertura boscosa de Costa Rica, pero a su vez es la región menos conocida del país, la que cuenta con el menor número de localidades de recolectas y menor cantidad de estudios de mamíferos .

\section{Conservación}

Costa Rica como primera respuesta a la problemática ambiental, desde que se estableció el sistema de Parques Nacionales en la década de 1970, ha dedicado gran parte de su territorio a la conservación, cerca del $11 \%$ del territorio corresponde a Parques Nacionales, y aproximadamente un $12 \%$ adicional cuenta con algún grado de protección; esto sin contar con las reservas biológicas privadas.

Desafortunadamente, en menos de cincuenta años, la cobertura boscosa de Costa Rica ha disminuido de un 80 a un $20 \%$, debido principalmente a la deforestación provocada con fines agrícolas y ganaderos (Bergoeing, 1998); en los ultimos años estas actividades han disminuido en su productividad y algunas áreas se han regenerado, muchas de ellas ahora son aprovechadas para turismo ecológico, o simplemente se han abandonado y presentan bosques secundarios; sin embargo la deforestación ha continuado. La tasa de deforestación estimada para Costa Rica es de $450 \mathrm{~km}^{2}$ por año, y un $70 \%$ del territorio está fuera de cualquier área protegida. Según Sánchez-Azofeifa et al.(2001), este proceso de deforestación tropical ha eliminado casi por completo los bosques húmedos tropicales y húmedos premontanos de Costa Rica.

Es precisamente la pérdida de hábitat debida a la deforestación el principal problema para la conservación de los mamíferos en este país, así como también son críticos los problemas de cacería (muchas veces muy cerca o dentro de áreas protegidas), y la contaminación del medio principalmente la del agua dulce.

Los esfuerzos por conservar la diversidad biológica del país deben consolidarse y son aún incipientes, si se considera que en los próximos 30 años la población humana de Costa Rica podría crecer de los cuatro millones de habitantes, que hay actualmente, a cerca del doble.

El futuro es aún más incierto para los mamíferos marinos, para los cuales los esfuerzos de conservación y manejo se han iniciado hasta hace muy pocos años. La falta de documentación sobre el estado de sus poblaciones, en conjunto con la muerte incidental en redes de pesca artesanal (Palacios y Gerrodette, 1996), la caza ilegal, la pérdida de habitat (Reinolds et al., 1995), la industria turística (Cubero, 2001) y la contaminación, han venido afectando las poblaciones residentes de varias especies. 
Las siguientes especies de mamíferos en Costa Rica pueden considerarse con mayores problemas de conservación. Se indica para cada una, las causas más próximas que pueden afectar su situación en el país. La conservación de los mamíferos en Costa Rica depende del fortalecimiento de las áreas protegidas ya existentes, del desarrollo de conocimiento biológico en el campo de la mastozoología y del control efectivo de la cacería, como se discute a continuación.

\section{Mamíferos voladores}

Hutson et al. (2001), en su evaluación mundial de microquirópteros, destacan la importancia de la región mesoamericana y publican la lista de UICN, donde prácticamente todas las especies de Costa Rica tienen alguna categoría de conservación, la gran mayoría como Bajo Riesgo, sin embargo Bauerus $\mathrm{d}$ ubiauquercus y Lasiurus castaneus sobresalen como especies Vulnerables. Ambas especies son raras en el país y son conocidas únicamente en Monteverde y sus alrededores (LaVal y Rodríguez-H, 2002).

Arita y Ortega (1998) evaluaron la riqueza de especies de quirópteros en Mesoamérica y, como resultado de este estudio, proponen que las especies presentes en Costa Rica que se destacan con la categoría de Estado Crítico son: Vampyressa nymphaea y Lasiurus castaneus, por ser de distribuciones restringidas y con abundancia escasa. Ectophylla alba, Hylonycteris underwoodi, Sturnira mordax y Myotis elegans, como especies de Estado Especial, por sus distribuciones, sus tipos de dormitorios o por niveles bajos de población.

Según Rodríguez-H et al. (2002), quienes evaluaron el estado de conservación de los murciélagos de Costa Rica con datos exclusivos del país, once especies: Peropteryx macrotis, Pteronotus gymnonotus, Chrotopterus auritus, Micronycteris minuta, Glyphonycteris daviesi, Trinycteris nicefori, Vampyrum spectrum, Lichonycteris obscura, Furipterus horrens, Bauerus dubiaquercus y Lasiurus castaneus, presentan una condición de Alto Riesgo de desaparecer de Costa Rica. Para todas ellas se recomienda generar mayor información acerca de su biología por medio de la investigación, ya que son poco conocidas $\mathrm{y}$, de esta forma, desarrollar políticas a nivel nacional para su conservación.

\section{MAMÍFEROS NO VOLADORES}

\section{Especies con mayor amenaza:}

Cyclopes didactylus, llamadas ceibitas. No existe información ni estudios en Costa Rica de esta especie. Por su ser arborícolas la deforestación representa el mayor peligro. Habitan zonas bajas húmedas de ambas vertientes. 
Myrmecophaga tridactyla, llamado oso caballo, muy probablemente es una especie extirpada en todo el país, pues no existen poblaciones conocidas de esta especie en, por lo menos, los últimos veinte años.

Saimiri oerstedii, conocido como mono ardilla o mono tití. Su principal problema es el comercio ilegal de mascotas. Aunque quedan poblaciones importantes en las áreas protegidas del Pacifíco sur y otras en areas de difícil acceso en el Pacífico Central, deben desarrollarse más estudios de campo sobre esta especie para conocer mejor el estado de sus poblaciones y el impacto de su extracción.

Ateles geoffroyi, llamado mono colorado, cuenta con poco conocimiento, y es muy vulnerable a la deforestación y contaminación de su hábitat. La cacería de esta especie también puede representar un peligro a corto plazo pero no se cuenta con datos suficientes.

Herpailurus yaguaroundi, Leopardus pardalis, Leopardus tigrinus, Leopardus wiedii, Puma concolor y Panthera onca son las seis especies nativas de felinos en Costa Rica. Todos estas especies depredadoras son muy vulnerables ante la pérdida de hábitat, en particular las especies de mayor tamaño que son P.concolor y P.onca, pues sus poblaciones requieren de mayor área y, en general, todas han sido muy perseguidas por su piel. Con excepción de P.onca, los felinos en Costa Rica podrían considerarse bien protegidos, por lo pronto, en los parques nacionales existentes, aunque se tiene muy poca información sobre ellos en el país y hacen falta estudios concretos. El caso del jaguar es más crítico y podría llegar a ser una especie extirpada si las tendencias actuales de cacería (incluyendo la cacería de sus presas) y la pérdida de hábitat fuera de áreas protegidas continúan (ver Sáenz y Carrillo, 2002; Vaughan y Temple, 2002). El proyecto del Corredor Biológico Mesoamericano y la educación ambiental representan las mejores estrategias para conservar al jaguar en este país.

Tapirus bairdii, llamada localmente danta. Se tiene poca información sobre esta especie en el país, son necesarios más estudios y, como en otros mamíferos grandes, la pérdida de hábitat y la cacería son los principales problemas de conservación de esta especie en Costa Rica.

Tayassu pecari, el cariblanco o chancho de monte, es otra especie que puede llegar a considerarse extirpada del país en los próximos años. El principal problema para su conservación es la cacería, incluso dentro de las áreas protegidas donde esta especie se encuentra. 
Cuniculus paca. El tepezcuintle es, con mucho, la especie más codiciada por los cazadores de Costa Rica, y el estado de sus poblaciones se desconoce. Los proyectos ya existentes para su crianza y aprovechamiento en cautiverio deberían ser la mejor opción para su conservación. También es necesario un plan específico de educación ambiental para esta especie, con mayor divulgación sobre su ecología e importancia sociocultural, pues a diferencia de otras especies que son más bien perseguidas por su piel, la cacería del tepezcuintle se da por su carne. Tiene también un componente cultural muy importante en este país, que podría ser mejor aprovechado para revertir las tendencias negativas sobre las poblaciones silvestres.

Trichechus manatus, el manatí, es el único mamífero marino de Centroamérica en la lista de condiciones críticas de UICN, punto de vista que es compartido por la mayoría de los científicos (Reynolds et al., 1995; Mou-Sue et al.,1990). Hábitats favorables aún existen a lo largo de la costa noreste y sur este (eg. Parque Nacional Tortuguero) del mar Caribe nacional, pero aún en esas áreas los manaties son raramente observados (Reynolds et al., 1995; Reynolds et al., 1999; Smethurst y Nietschmann, 1999). Esta especie fue abundante en la costa noreste de Costa Rica, particularmente durante 1940's su número decreció dramáticamente como resultado de la caza ilegal, redes de pesca y degradación de hábitat, y desde los años 1970's raramente se les encuentra (Mou-Sue et al., 1990).

\section{AGRADECIMIENTOS}

Agradecemos al Dr. Gerardo Ceballos por motivarnos para hacer este trabajo. A Joaquín Arroyo-Cabrales y Ragde Sánchez Talavera por sus comentarios al manuscrito. A Richard K. LaVal por su colaboración durante la elaboración del artículo. F. A. Chinchilla agradece el apoyo del Instituto de Ecología (UNAM), el Proyecto Corredor Biológico Mesoamericano (WWF-Centroamérica) y Idea Wild durante la realización de este trabajo.

\section{LITERATURA CITADA}

Acevedo-Gutiérrez, A. 1996. Lista de mamíferos marinos en Golfo Dulce e Isla del Coco, Costa Rica. Revista de Biología Tropical, 44 (2):933-934.

Alfaro, A. 1897. Mamíferos de Costa Rica. Tipografía Nacional, San José Costa Rica.

Alston, E.R. 1879. Biologia Centrali-Americana. London, Mammalia, Vol 1, xx+220, 22pls Arita, H. y J. Ortega. 1988. The Middle American Bat Fauna: Conservation in the NeotropicalNeartic Border. Pp XXXXX en: Bat Biology and Conservation (T. H. Kunz y P. Racey eds). Smithsonian Institution Press. 
Arroyo-Cabrales, J. y R. D. Owen. 1997. Enchisthenes hartii. Mammalian Species, 546:1-4.

Baker, R. J., C. A. Porter, J. C. Patton y R. A. Van Den Bussche. 2000. Systematics of bats of the family Phyllostomidae based on RAG2 DNA sequences. Occasional Papers, Museum of Texas Tech University, 202:1-16.

Baker, R.J., S. Solari y F.G. Hoffmann. 2002. A New Central American Species from The Carollia brevicauda Complex. Occasional Papers, Museum of Texas Tech University, 217:1-12.

Bergoeing, J.P. 1998. Geomorfología de Costa Rica. Instituto Geográfico Nacional. Costa Rica.

Carrillo, E, G. Wong y J. Sáenz. 2000. Mamíferos de Costa Rica. Instituto de Biodiversidad, San José, Costa Rica.

Ceballos, G.C., J. Arroyo-Cabrales and R. Medellín. 2002. The mammals of Mexico: composition, distribution, and conservation. Occasional Papers, Museum of Texas Tech University, 218:1-27.

Cubero-Pardo, P. 2001. Assessing the impact of whale-watching activities on wild cetaceans in Costa Rica. $14^{\text {th }}$ Biennial Conference on The Biology of Marine Mammals. Vancouver B. C., Canada.

Dragoo, J. W., y R. L. Honeycutt. 1997. Systematics of mustelid-like carnivores. Journal of Mammalogy, 75:266-276.

Engstrom, M.D. y B.K. Lim. 2002. Mamíferos de Guyana. Pp: 329-375, en: Diversidad y Conservación de los Mamíferos Neotropicales (G. Ceballos y J. A. Simonetti, eds.) CONABIO-UNAM, México D. F.

Frantzius, A.V. 1869. Los Mamíferos de Costa Rica. Traducido por D.R. Cortéz. 1963. Revista de los Archivos Nacionales de Costa Rica. Brenesia, 39/40:179-180.

Goodwin, G.G. 1946. Mammals of Costa Rica. Bulletin of the American Museum Natural History, 87: 271-473

Grooves, C. 2001. Primate Taxonomic. Smithsonian Institution Press, Washington, D. C.

Handley, C. O., Jr. 1987. New species of mammals from northern south America: fruit-eating bats, genus Artibeus Leach. Pp. 163-172, en: Studies in Neotropical mammalogy, essays in honor of Philip Hershkovitz, (B. D. Patterson y R. M. Timm, eds). Fieldiana Zoology New Series.

Harcourt, C. S. y J. A. Sayer. 1996. Conservation Atlas of Tropical Forests: The Americas. UICN-CIFOR-WCMC-BP. Simon \& Shuster. New York.

Harris Jr., W.P. 1932. Four new mammals from Costa Rica. Occasional Papers, Museum of Zoololgy University of Michigan,476:1-15.

Hershkovitz, P. 1992. The South America genus Gracilinanus Gardner and Creighton, 1989 (Marmosidae, Marsupialia): a taxonomic review with notes on general morphology and relationships. Fieldiana Zoology New series, 70:1-56.

Hutson, A., S., Mickleburgh y P. Racey. 2001. Global Status Survey and Conservation Action Plan. Microchiropteran Bats. IUCN/SSC, Chiroptera Specialist Group, Gland and Cambridge.

Iudica, C. A. 2000. Systematic Revision of the neotropical Fruit Bats of the Genus Sturnira: A Molecular and Morphological Approach. Tesis Doctoral, Universidad de Florida, E.U.A. 
Janzen, D.H. 1983. Costa Rican Natural History. University of Chicago Press, Chicago.

LaVal, R.K. y B. Rodríguez-H. 2002. Murciélagos de Costa Rica. Editorial INBIO. San José, Costa Rica.

Leatherwood, S. y R. R. Reeves. 1983. The Sierra Club Handbook of Whales and Dolphins. Sierra Club, San Francisco, C.A.

Lee, T. E., Jr., S. R. Hoofer y R. A. Van Den Bussche. 2002. Molecular phylogenetics and taxonomic revision of the genus Tonatia (Chiroptera: Phyllostomidae). Journal of Mammalogy, 83(1):49-57.

López-González, C. y S. J. Presley. 2001. Taxonomic Status of Molossus bondae J. A. Allen, 1904 (Chiroptera: Molossidae), with description of a new subspecies. Journal of Mammology, 82(3):760-774.

McKenna, M. C. y K. Bell. 1997. Classification of mammals above the species level. Columbia University Press, New York.

Méndez, E. 1993. Los roedores de Panamá. Laboratorio Conmemorativo Gorgas, Panamá. Impresora Pacífico.

Mora J. M. 2000. Los Mamiferos Silvestres de Costa Rica. Editorial Universidad Estatal a Distancia. San José, Costa Rica.

Mou-Sue, L. Chen, D. H., Bonde, R. K. y T. J. O'Shea. 1990. Distribution and status of manatees (Trichechus manatus) in Panama. Marine Mammal Science, 6(3):234-241.

Owen, R. D. 1987. Phylogenetic analyses of the bat subfamily Stenodermatinae (Mammalia: Chiroptera). Special Publications, Museum, Texas Tech University, 26:1-65.

Palacios, D. M. y T. Gerrodette. 1996. Potential impact of artisanal gillnet fisheries on small cetacean populations in the eastern tropical Pacific. Administrative Report LJ-96-11. National Marine Fisheries Servies, SWFSC, La Jolla, CA 92038.

Peters S. L., B. K. Lim y M. D. Engstrom. 2002. Systematics of Dog-Faced bats (Cynamops) based on molecular and morphometric data. Journal of Mammology, 83(4):1097-1110.

Reynolds, J. E., W. A. Szelistowski y M. A. León. 1995. Status and conservation of manatees Trichechus manatus manatus in Costa Rica. Biological Conservation, 71:193-196.

Reynolds, J. E. III, D. K. Odell y S. A. Sommel. 1999. Marine Mammals of the World. Pp. 114. en: Biology of Marine Mammals (J. E. III Reynolds y S. A. Rommel, eds.). Smithsonian Institution Press, Washington and London.

Rodríguez-H., B. y D. E. Wilson. 1999. Lista y distribución de las especies de murciélagos de Costa Rica. Occasional Papers Conservation Biological, Conservation International, 9:1-34.

Rodríguez-H., B., G. Barrantes y R. K. LaVal. 2002. Evaluación del estado de conservación de las especies de murciélagos en Costa Rica. Mesoamérica. Vol. 6, No. 3.

Rodríguez-H, B., W. Pineda, M. Fernández y R. K. LaVal. 2003. First Record for the Bat Lasiurus intermedius H. Allen (Chiroptera: Vespertilionidae) from Costa Rica. Bat Research News. (en prensa).

Rodríguez, J. y F. A. Chinchilla. 1996. Lista de Mamíferos de Costa Rica. Revista Biología Tropical, 44:877-890.

Rodríguez-Fonseca, J. 2001. Diversidad y distribución de los cetáceos de Costa Rica (Cetacea: Delphinidae, Physeteridae, Ziphiidae y Balaenopteridae). Revista de Biología Tropical, 49 (2):135-143. 
Saenz, J. C. y E. Carrillo. 2002. Jaguares depredadores de ganado en Costa Rica: un problema sin solución?. Pp 127-137, en: El Jaguar en el Nuevo Milenio (R.A. Medellin et al., eds). México.

Sánchez-Azofeifa, G.A., R.C. Harriss y D. L. Skole. 2001. Deforestation in Costa Rica: A Quantitative Analysis Using Remote Sensing Imagery. Biotropica, 33(3):378-384.

Simmons, N. B. 1996. A New Specie of Micronycteris (Chiroptera: Phyllostomidae) from northeastern Brazil, with comments on phylogenetic relationships. American Museum Novitates, 3158:1-34.

Simmons, N. B. 1998. A reappraisal of interfamilial relationships of bats. Pp. 3-26, en: Bat Biology and Conservation (T. H. Kunz y P. A. Racey, eds.). Smithsonian Institution Press, Washington D. C.

Simmons, N. B. y C. O. Handley, Jr. 1998. A revision of Centronycteris Gray (Chiroptera: Emballonuridae) with notes of natural history. American Museum Novitates, 3239:1-28.

Simmons, N. B. y R. S. Voss. 1998. The mammals of Paracou, French Guiana: A Neotropical lowlands rainforest fauna. Part 1. Bats. Bulletin of the American Museum of Natural History, 237:1-219.

Smethurst, D. \& B. Nietschmann. 1999. The distribution of manatees (Trichechus manatus) in the coastal waterways of Tortuguero, Costa Rica. Biological Conservation, 89:267-274.

Timm, R. M. y R. K. LaVal. 1998. A field key to the bats of Costa Rica. Center of Latin American Studies, University of Kansas, Occasional Publications Series, 22:1-30.

Unión Mundial para la Naturaleza. 1999. Listas de Fauna de Importancia para la Conservación en Centroamérica y México. San José, Costa Rica.

Vaughan C. S. y S. Temple. 2002. Conservacion del Jaguar en Centroamérica. Pp 355-366, en: El Jaguar en el Nuevo Milenio (R.A. Medellin et al., eds). México.

Wetterer, A. L., M. V. Rockman y N. B. Simmons. 2000. Phylogeny of Phyllostomid bats (Mammalia:Chiroptera) data from diverse morphological systems, sex chromosomes, and restriction sites. Bulletin of the American Museum of Natural History, 248:1-200.

Wilson, D. E. 1983. Checklist of Mammals of Costa Rica. Pp. 443-447, en: Costa Rican Natural History (D. H. Janzen, ed.). University of Chicago Press, Chicago. Illinois.

Wilson, D. E., R. M. Timm, and F. A. Chinchilla. 2002. Mamíferos de Costa Rica. Pp. 227254, en: Diversidad y Conservación de los Mamíferos Neotropicales (G. Ceballos y J. A. Simonetti, eds.). CONABIO, México D. F.

Wilson, D. E. y D. M. Reeder (editores). 1993. Mammal species of the World, a taxonomic and geographic reference. $2^{\text {nd }}$ ed. Smithsonian Institution Press, Washington, D. C.

Whitmore, T. C. 1990. An Introduction to Tropical Rain Forests. Clarendon. Oxford.

Woodman, N. 2000. Cryptotis merriami Choate in Costa Rica: Synotopy with Cryptotis nigrescens (Allen) and possible character displacement (Mammalia:Insectivora). Caribbean Journal of Science, 36 (3-4):289-299.

Woodman, N. y R. M. Timm. 1993. Intraspecific an interspecific variation in the Cryptotis nigrescens Species Complex of Small-Eared Shrews (Insectivora:Soricedae), with the description of a New Species from Colombia. Fieldiana Zoology, 74:1-29.

Wright, D. B. 1989. Phylogenetic relationships of Catogonus wagneri: sister taxa from the Tertiary of North America. Pp 281-308, en: Advances in Neotropical Mammology (K. H. Redford y J. F. Eisenberg, eds.). Sandhill Crane Press, Gainsville, Florida. 


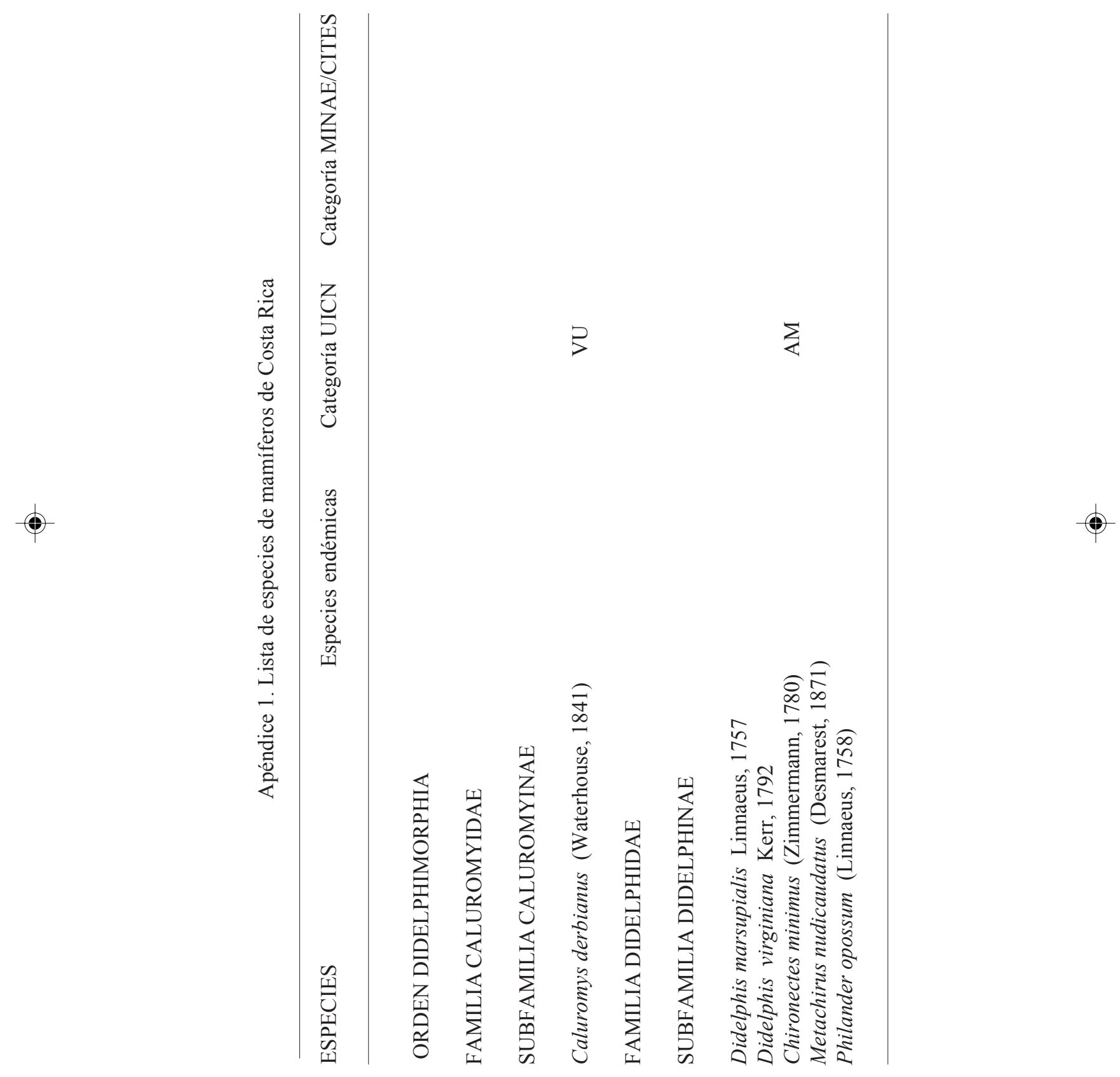


vol. 6

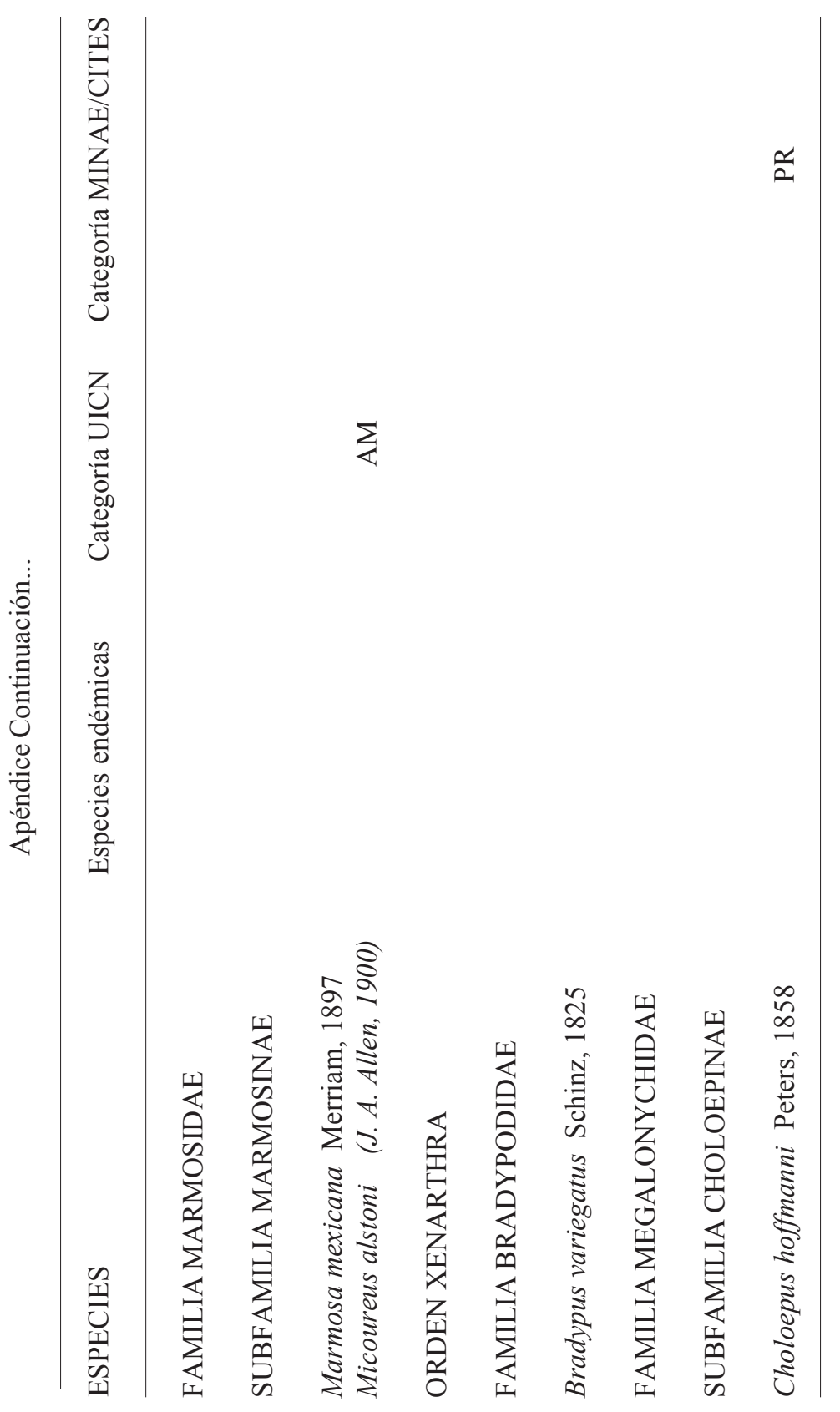




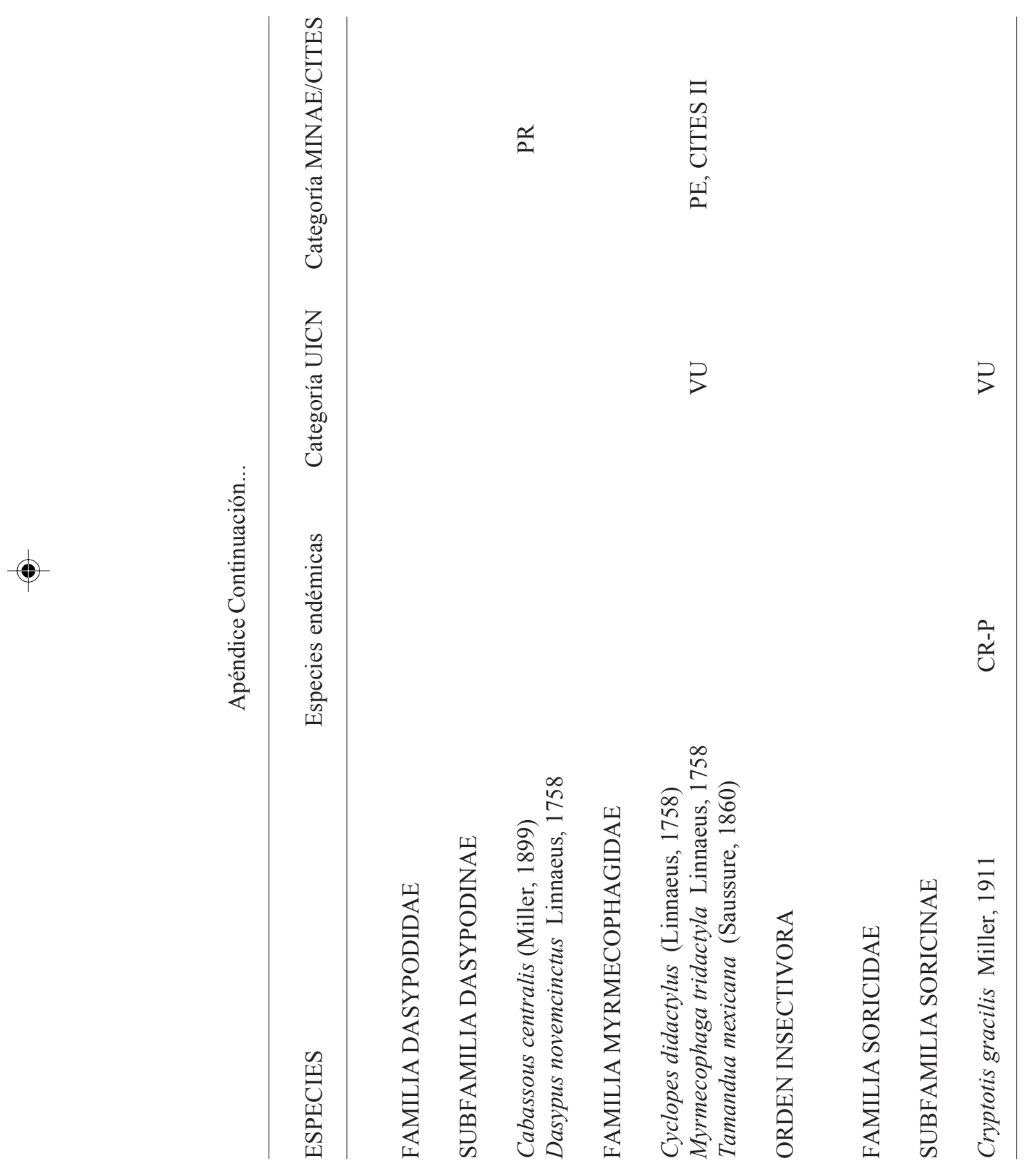




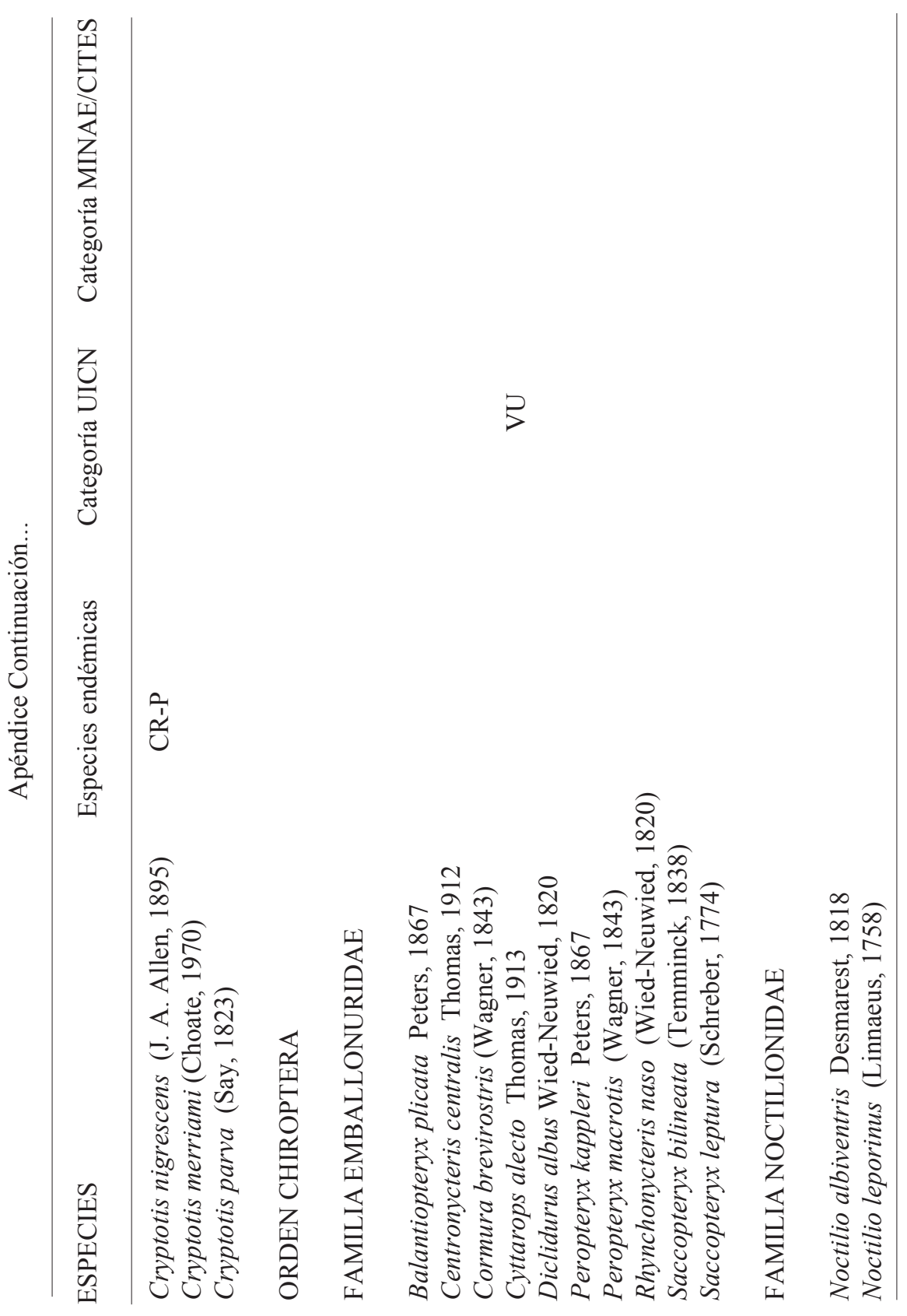




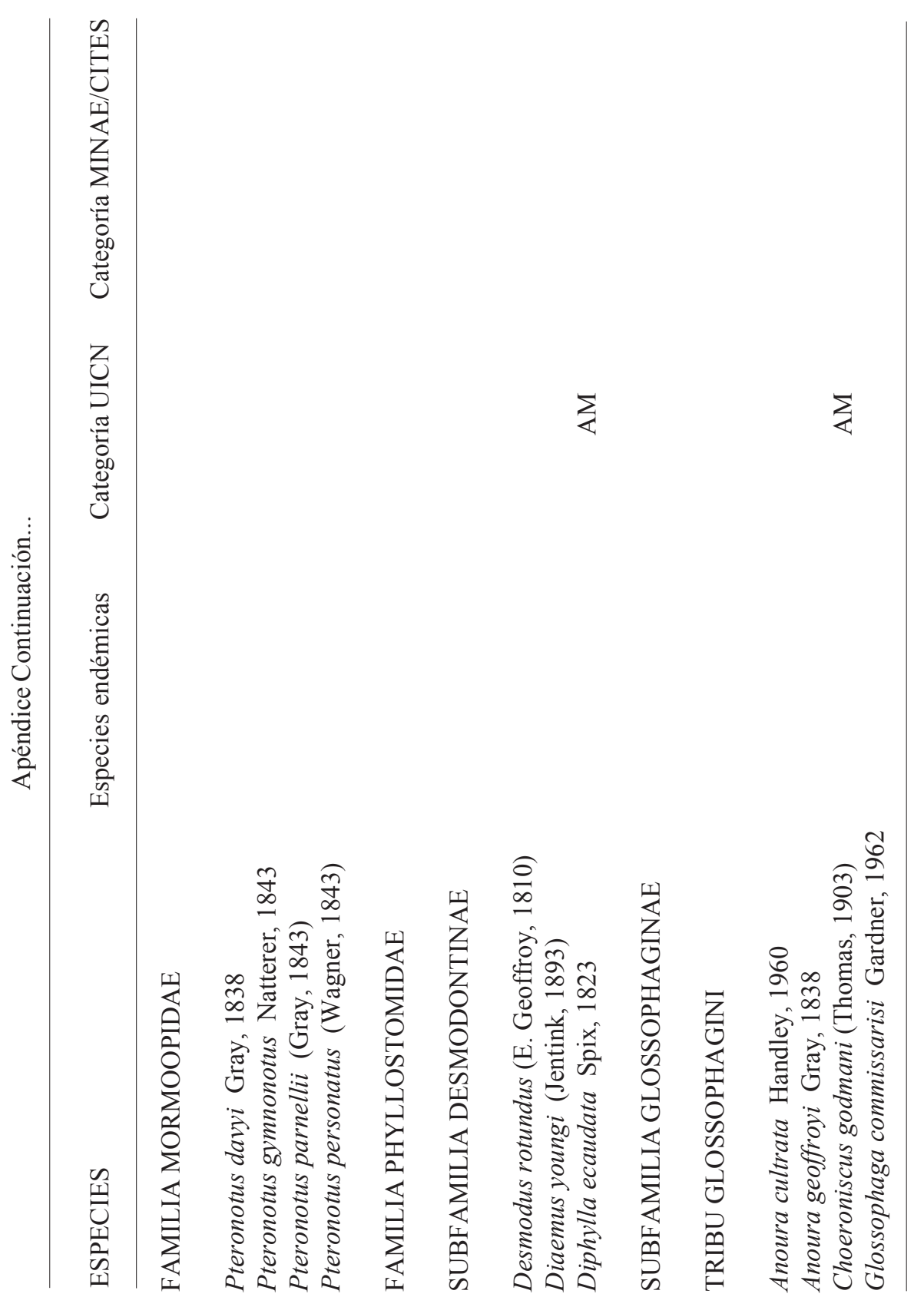


vol. 6

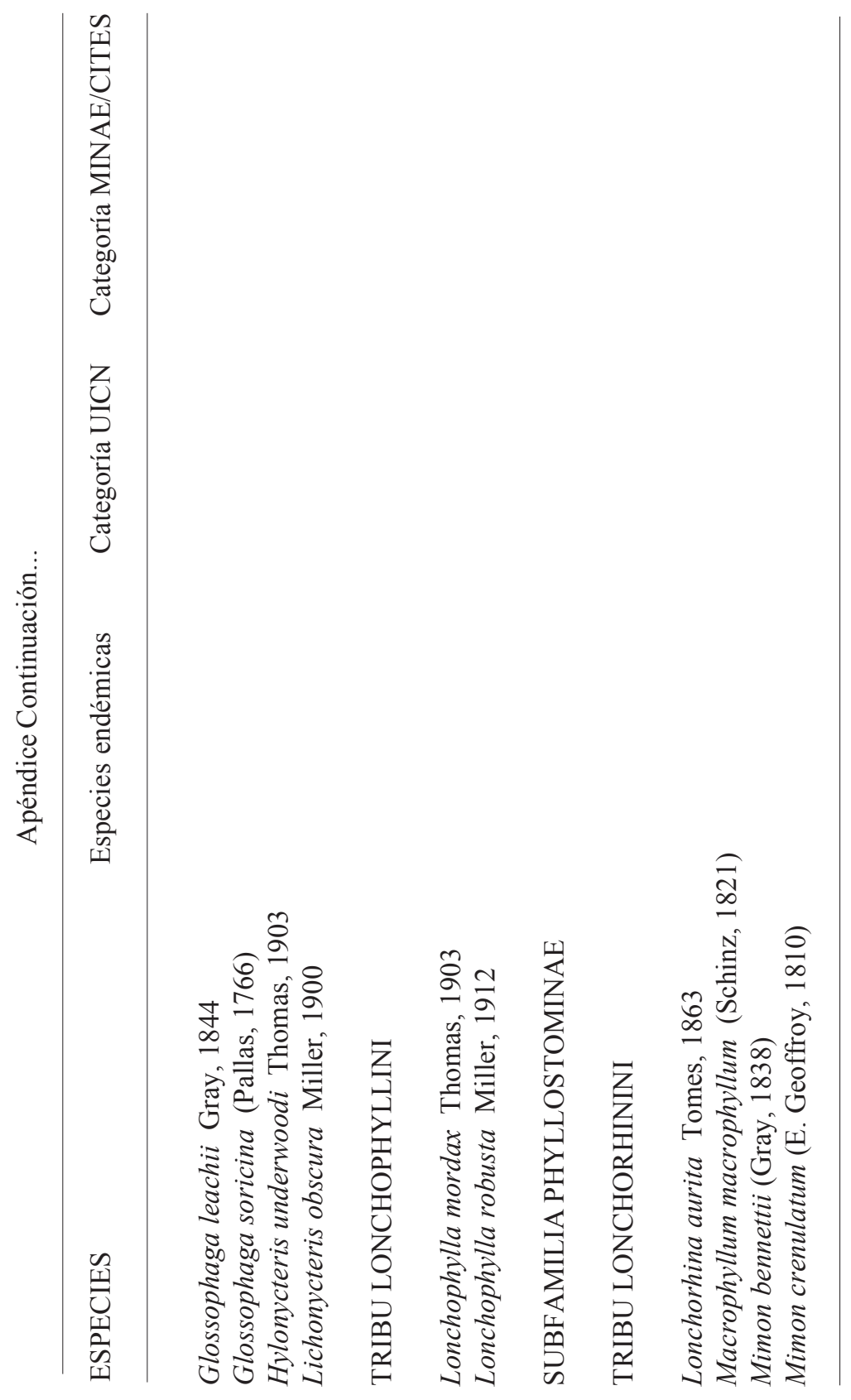




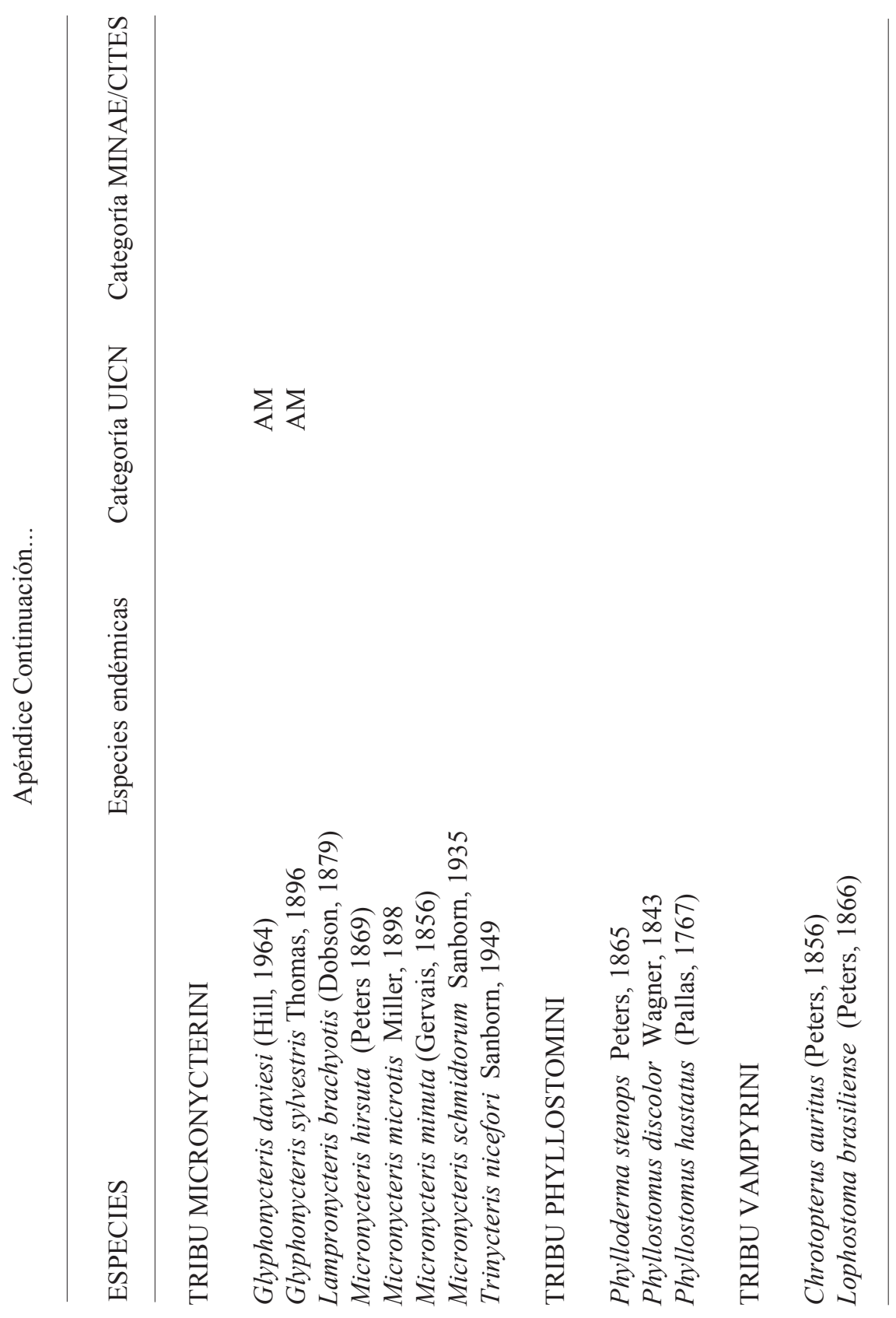




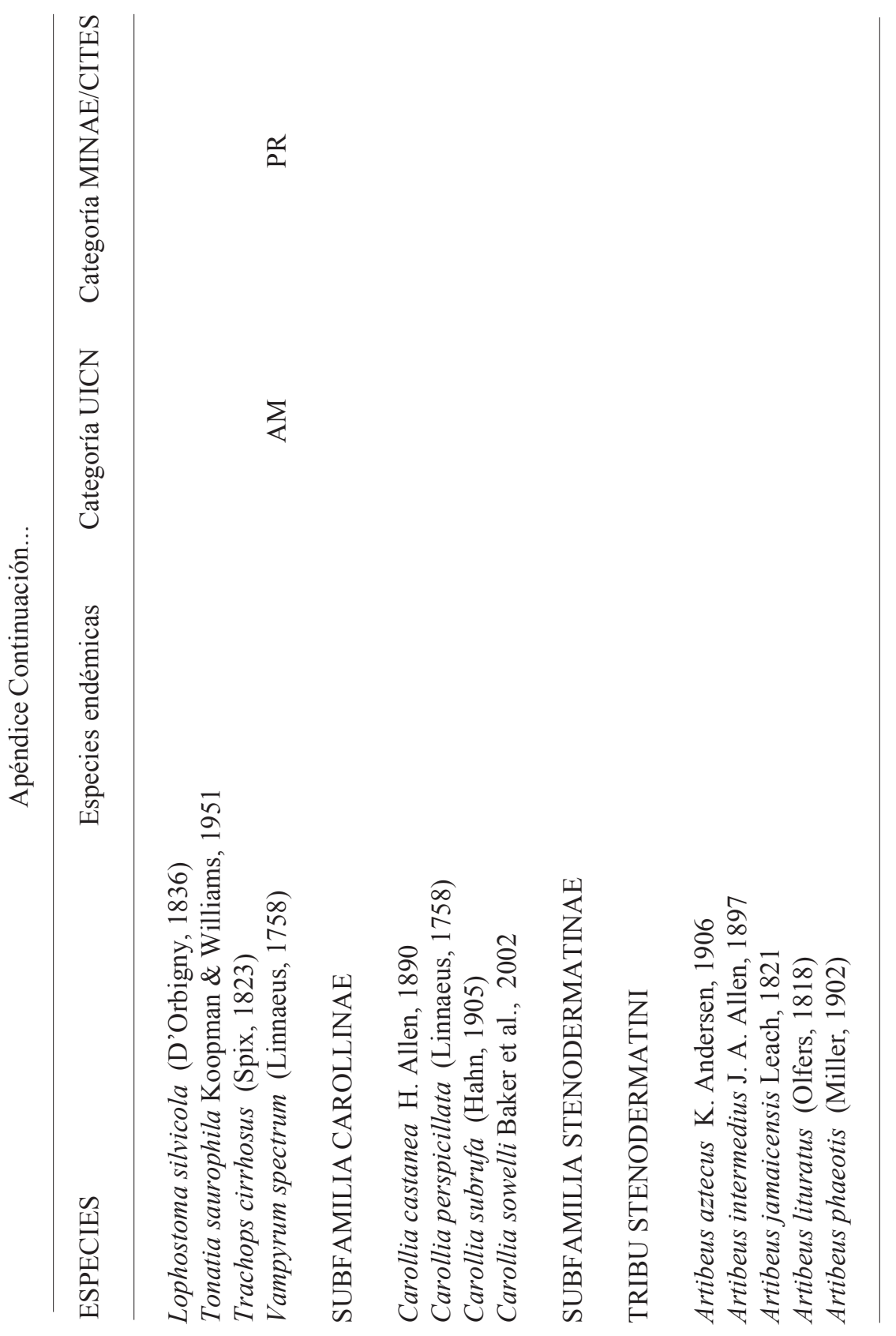




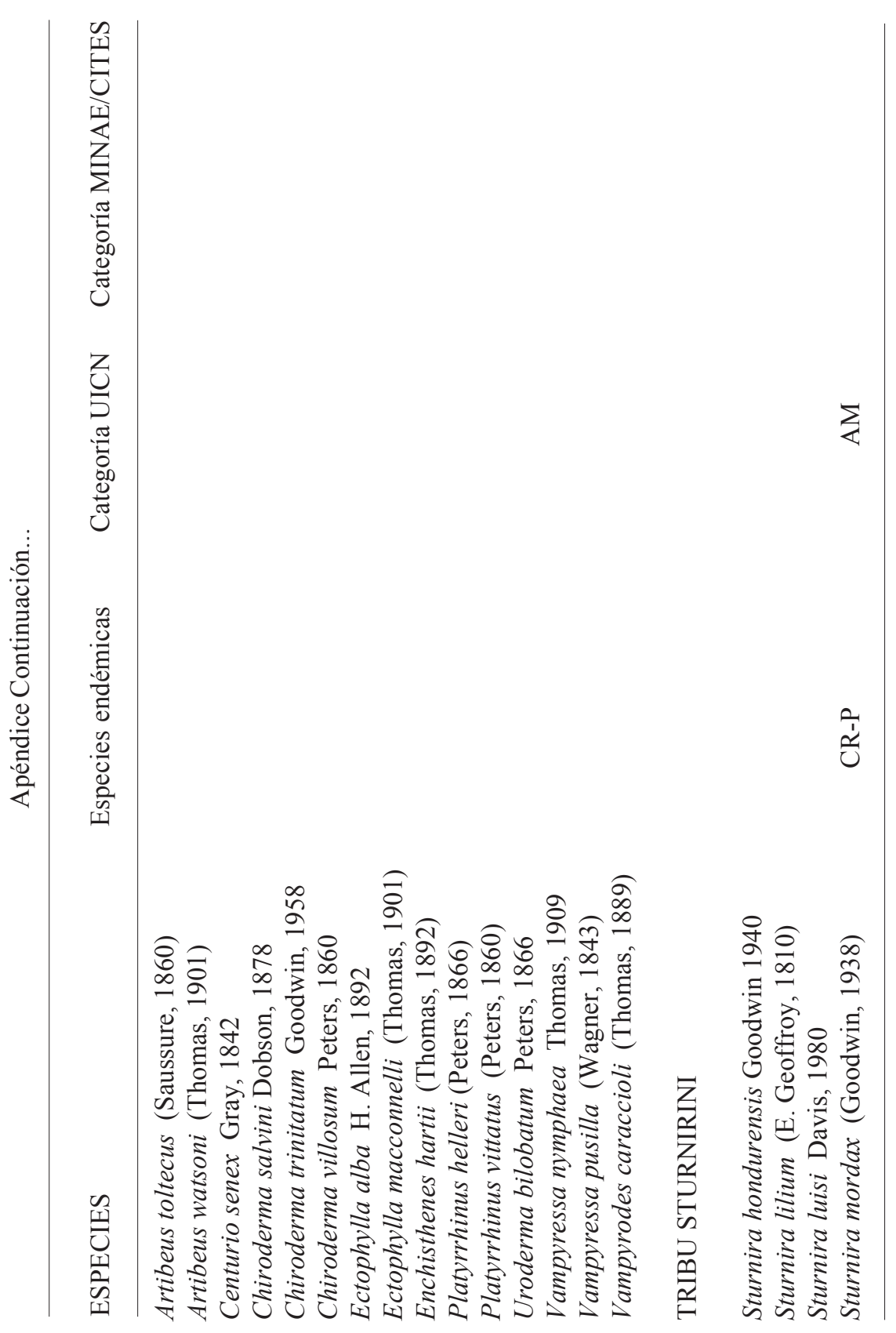




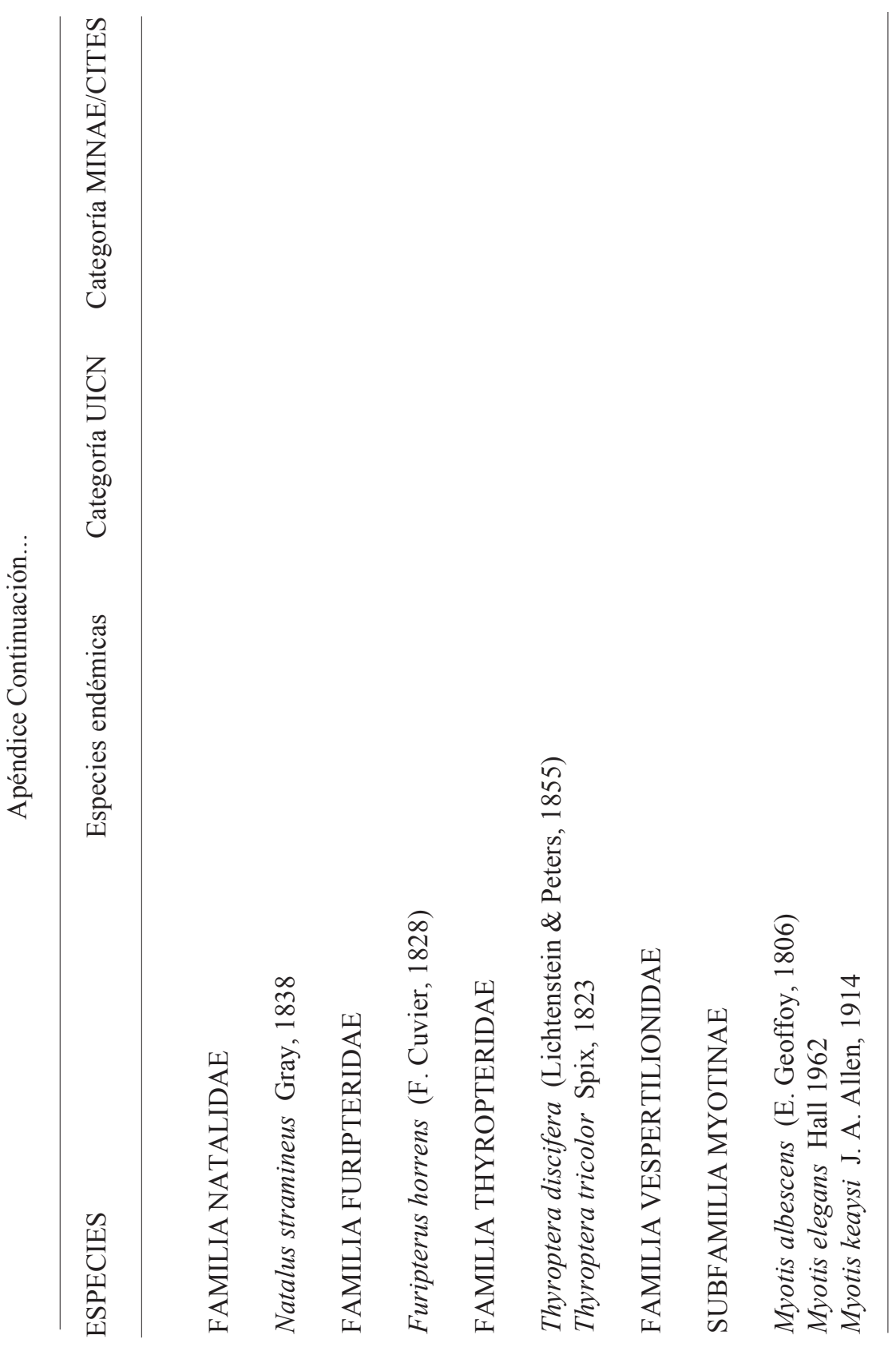




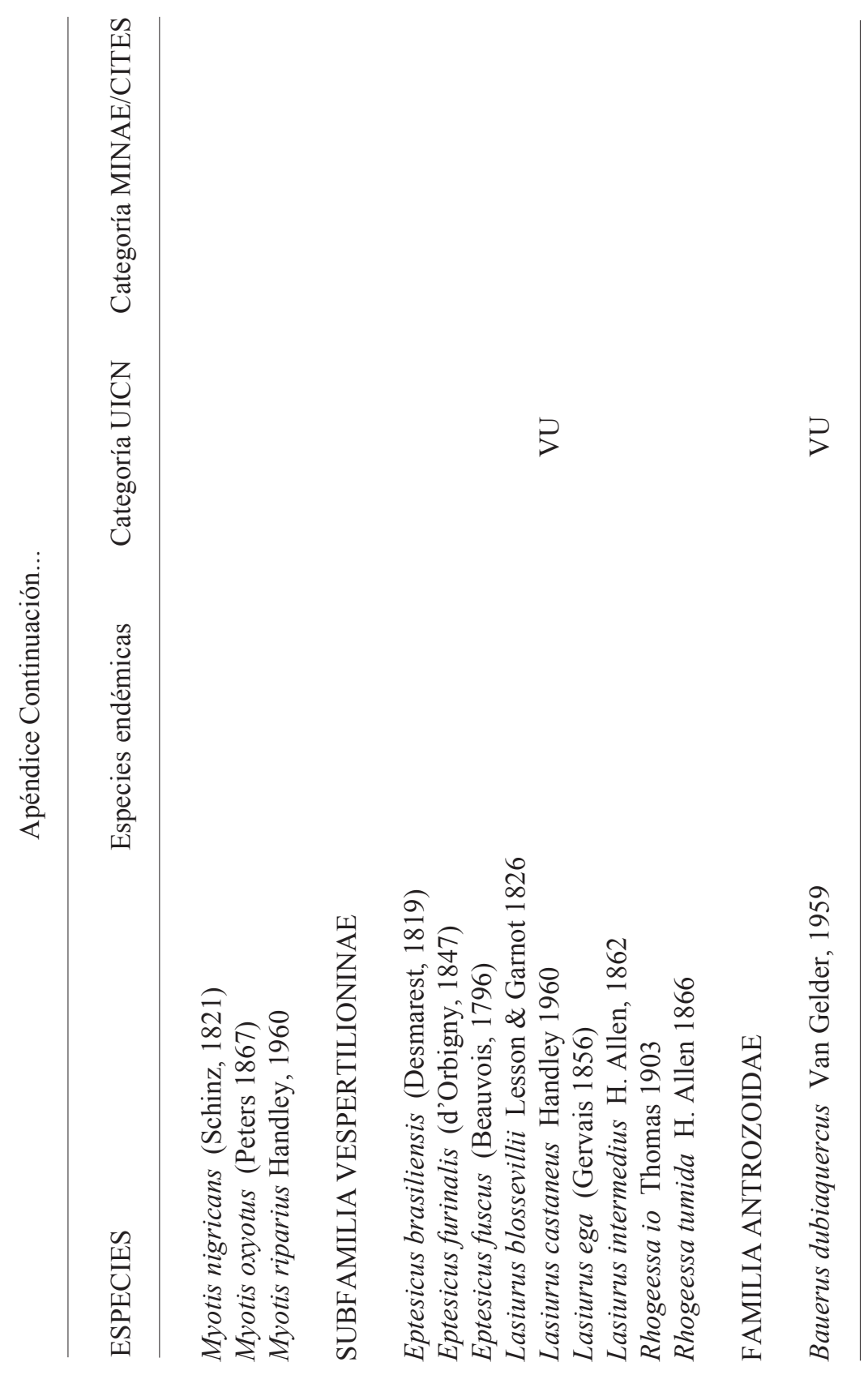


vol. 6

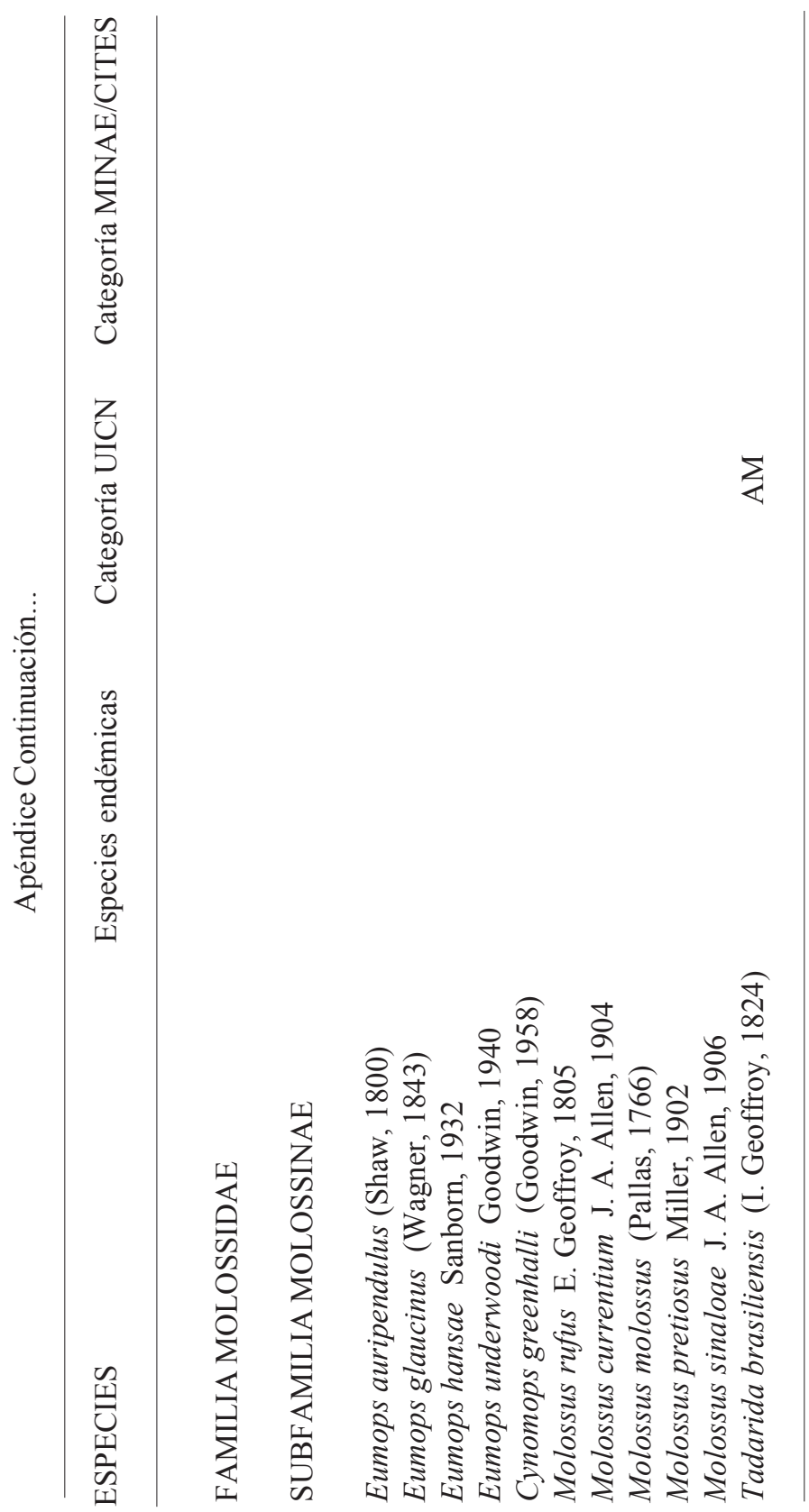




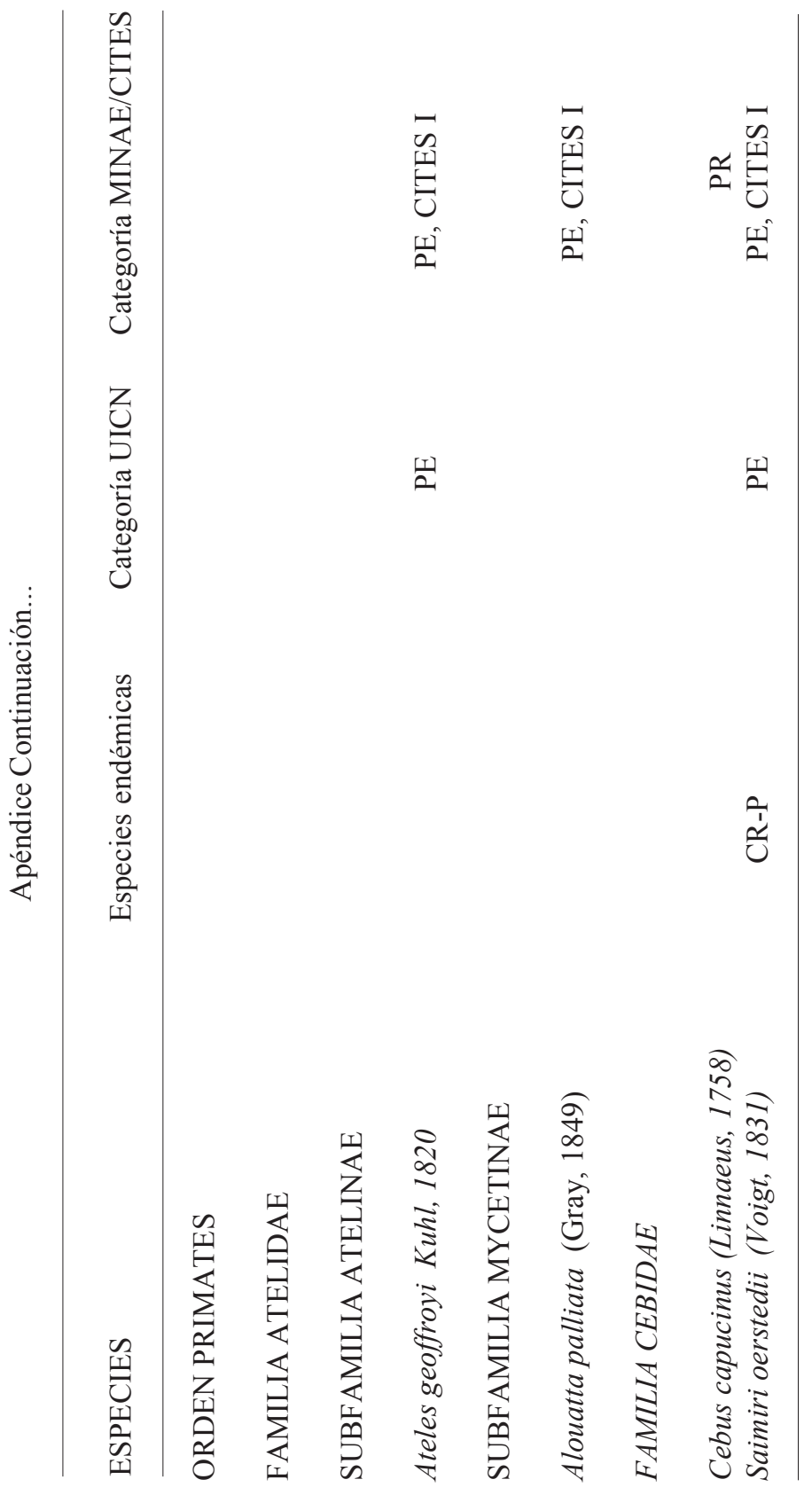


vol. 6

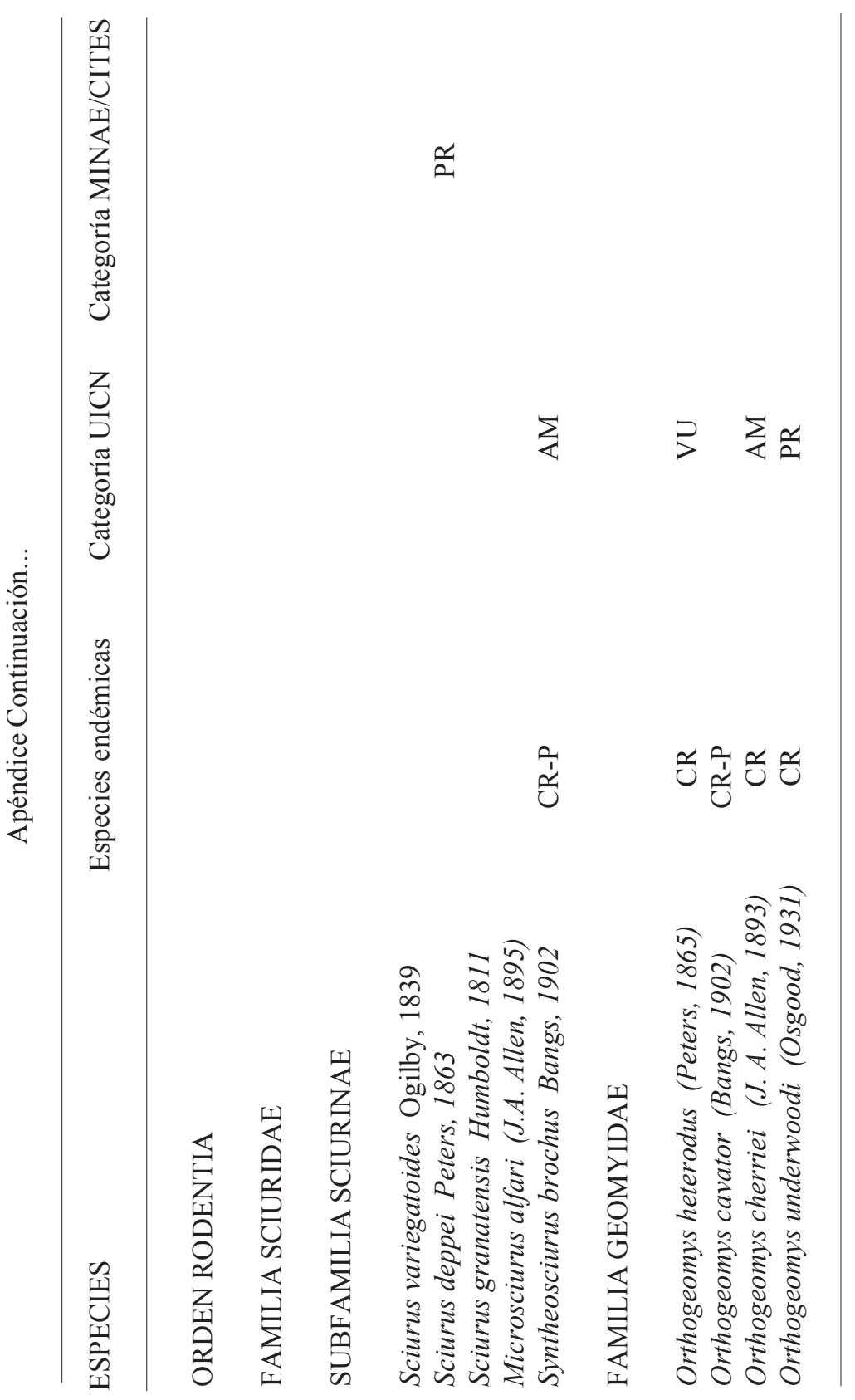




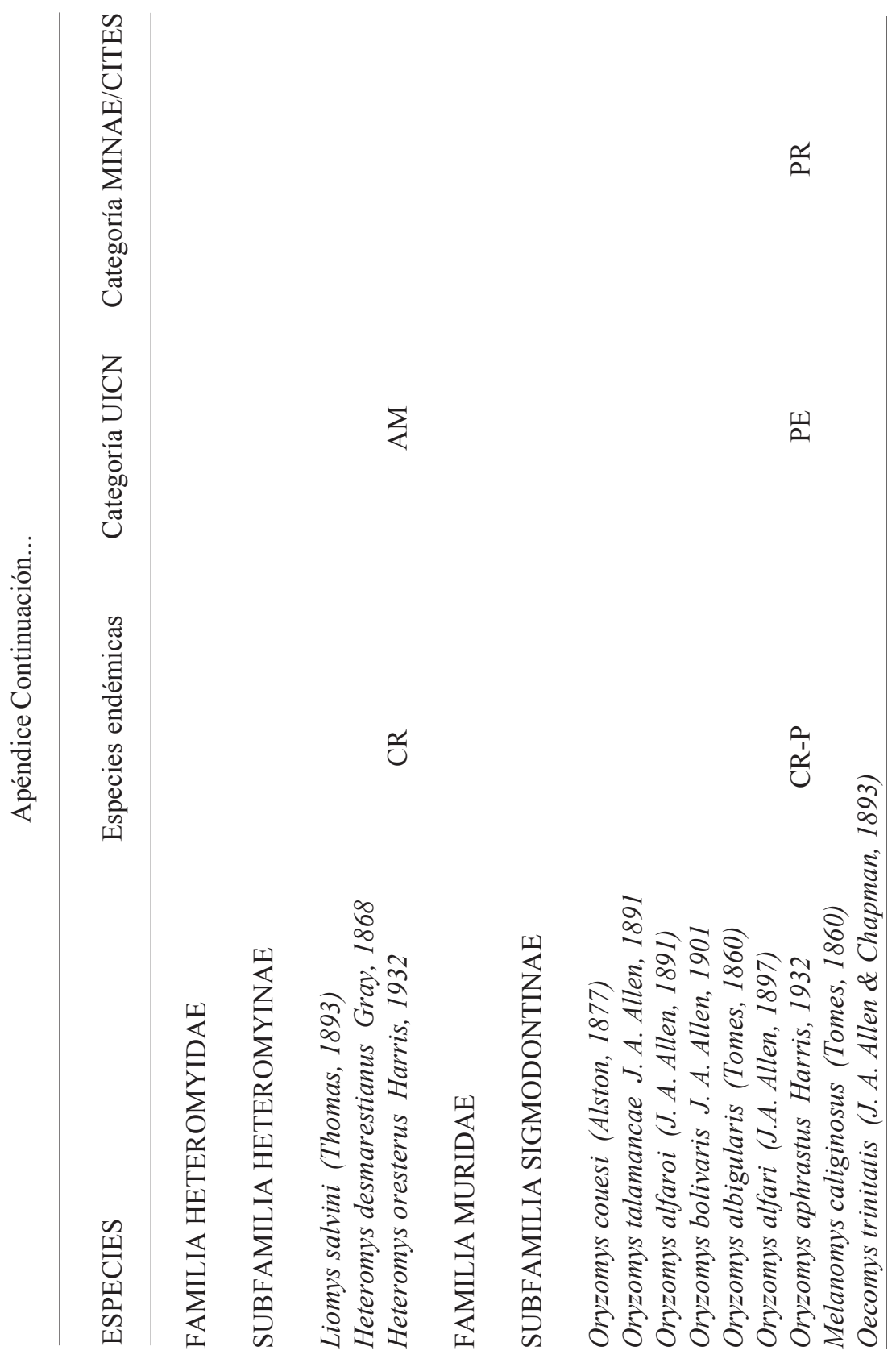




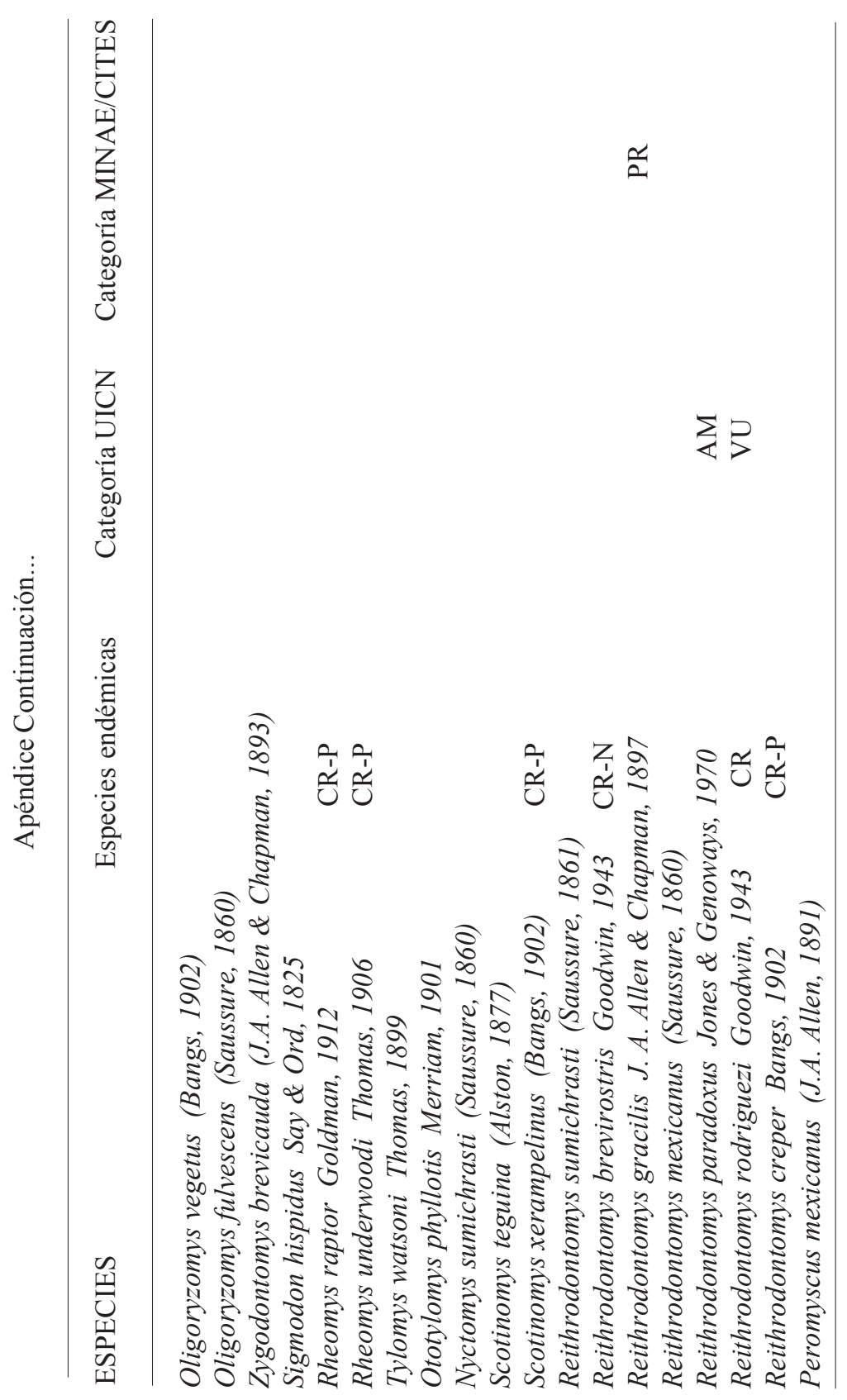




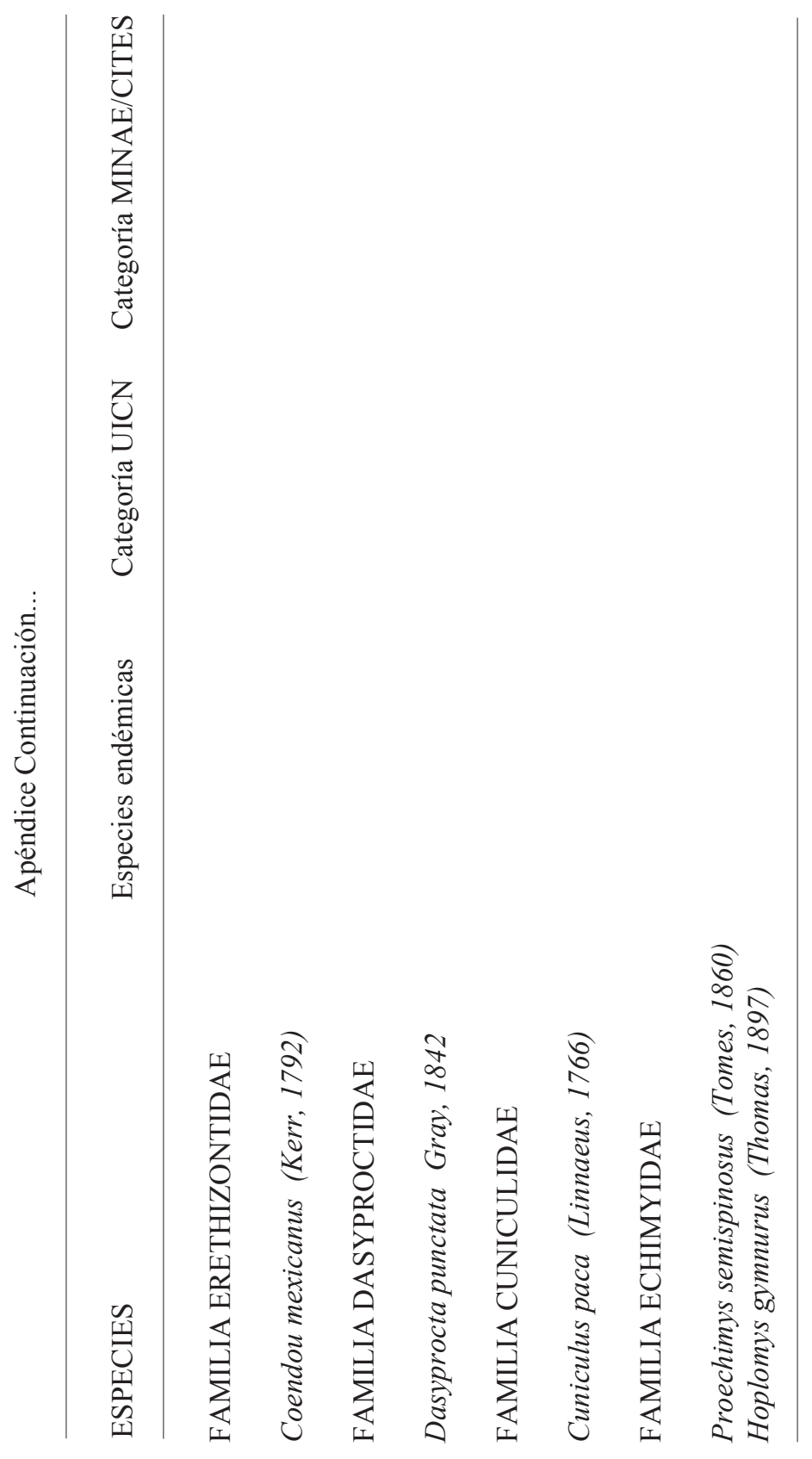




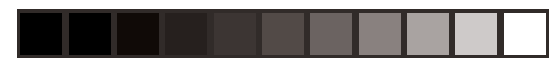

50
Revista Mexicana de Mastozoología

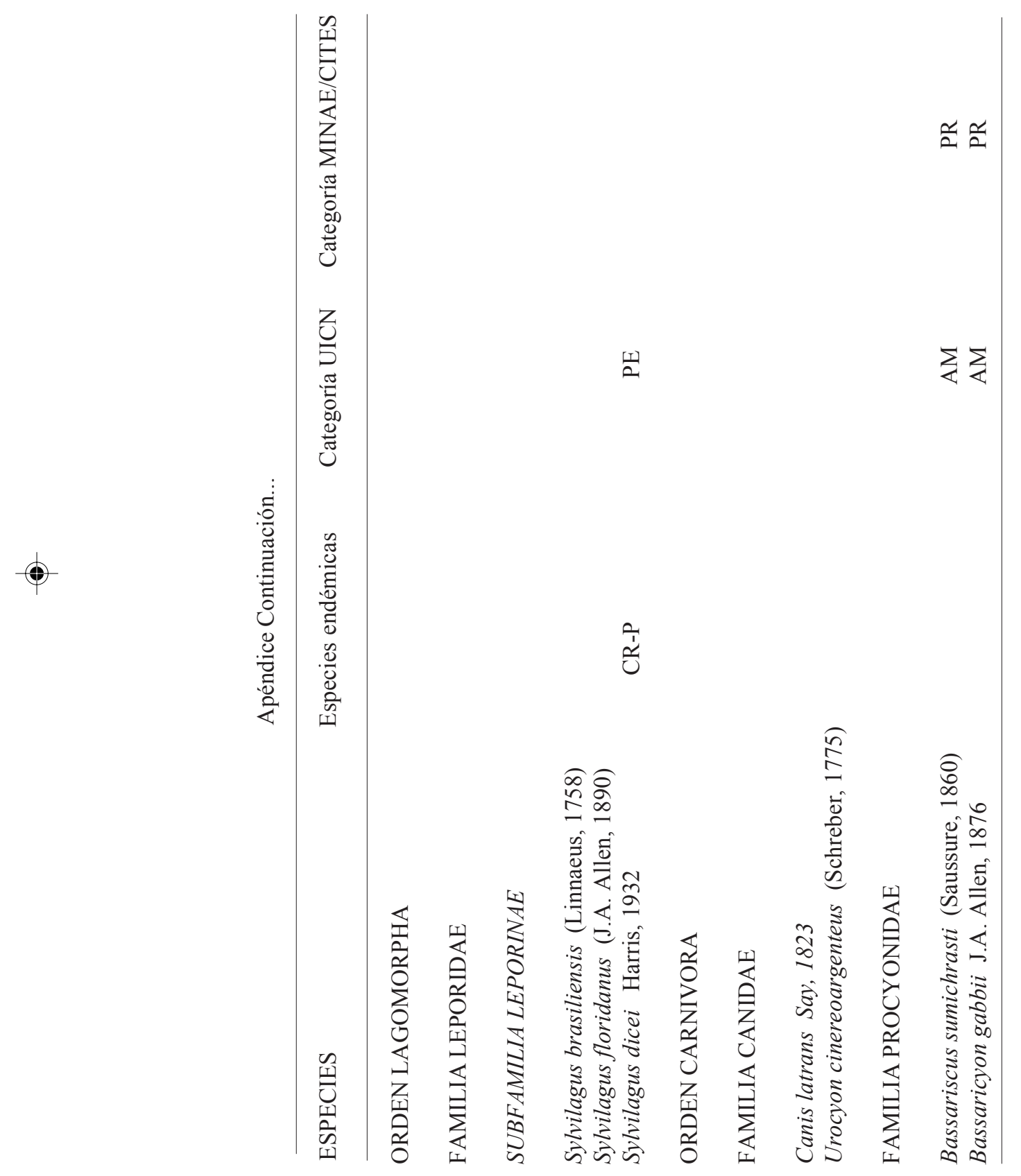

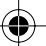

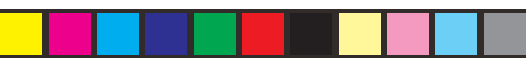

\begin{tabular}{|l|l|l|l|l|}
\hline$\square$ & $\square$ & $\square$
\end{tabular}

vol. 6 


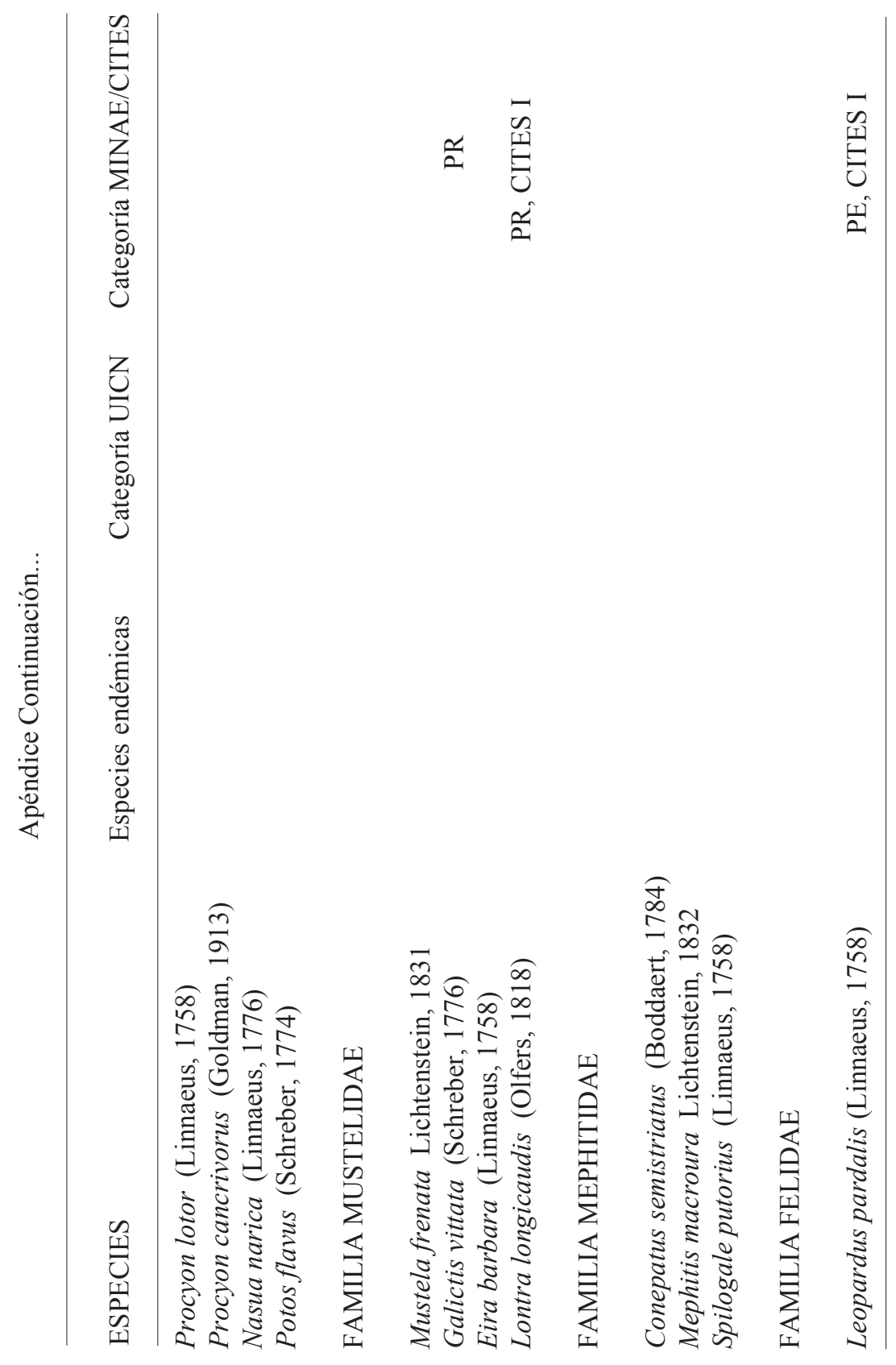




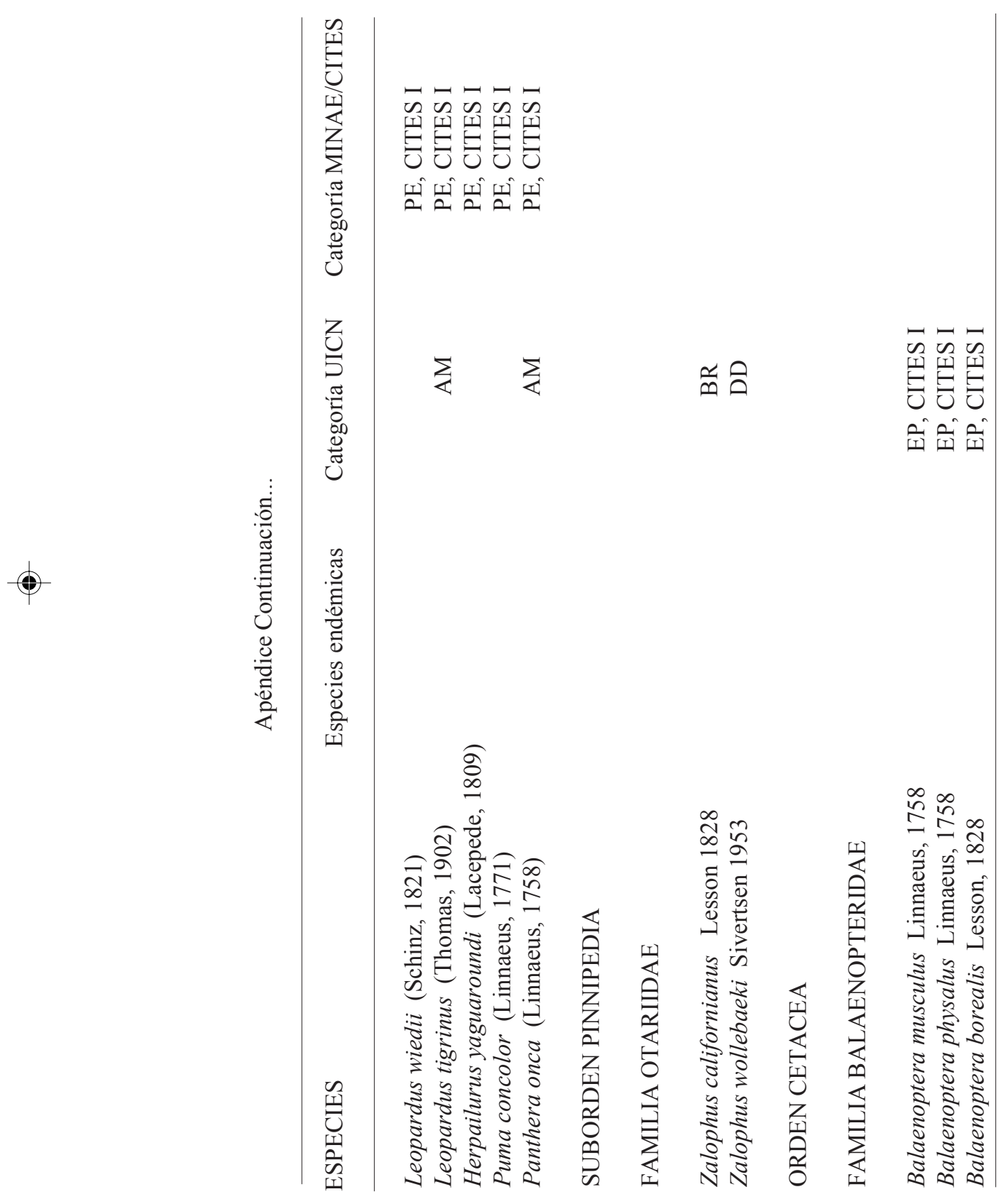




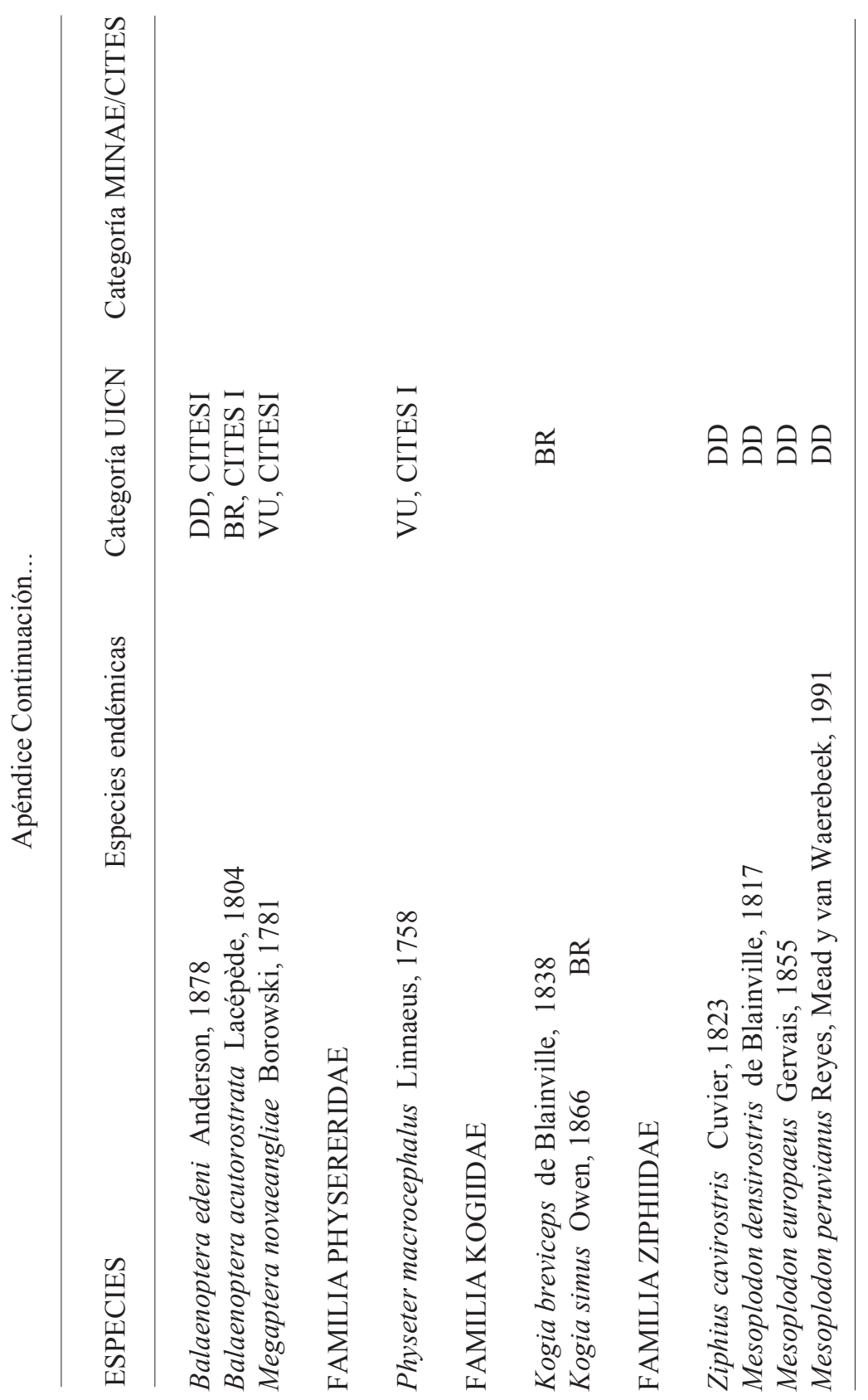




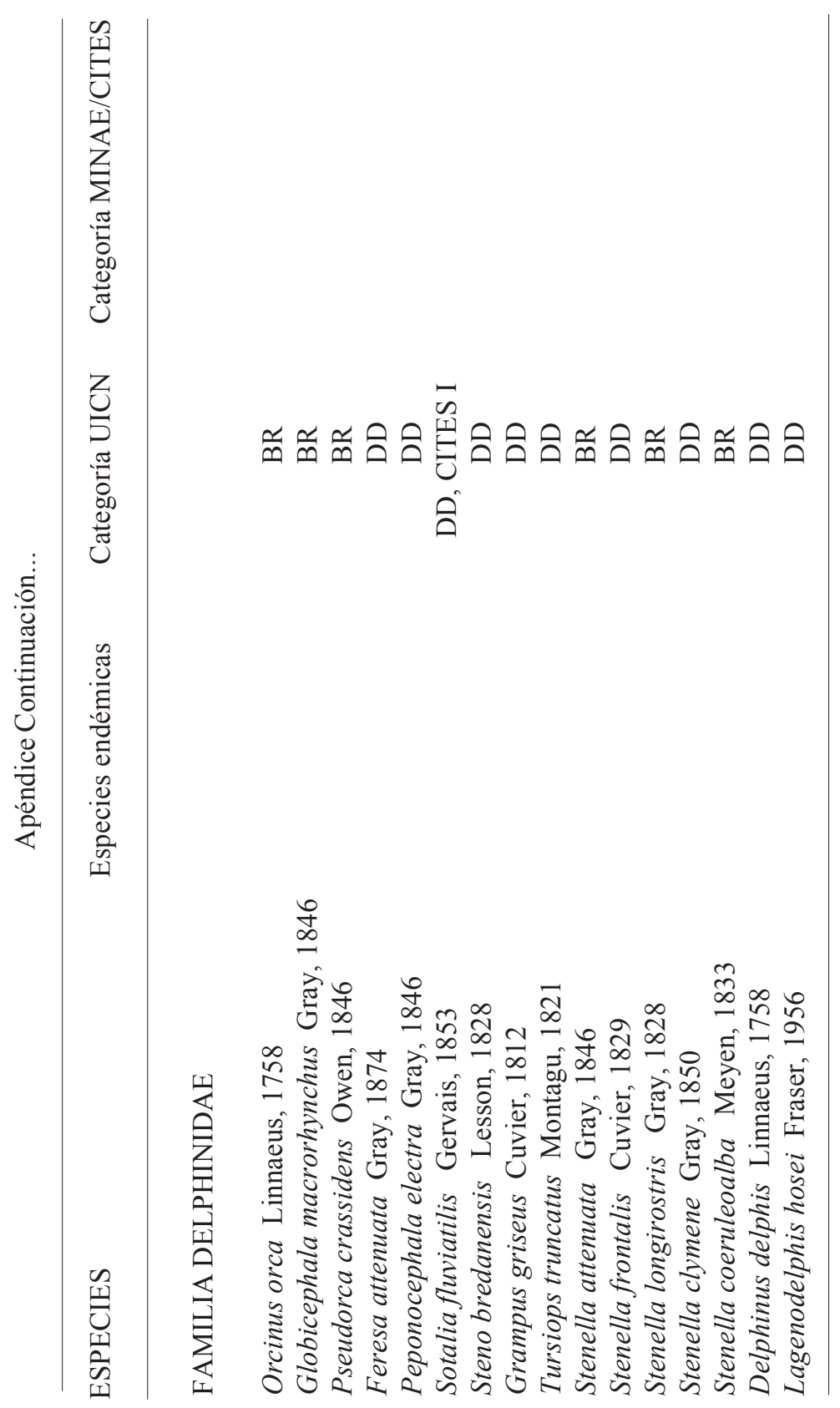




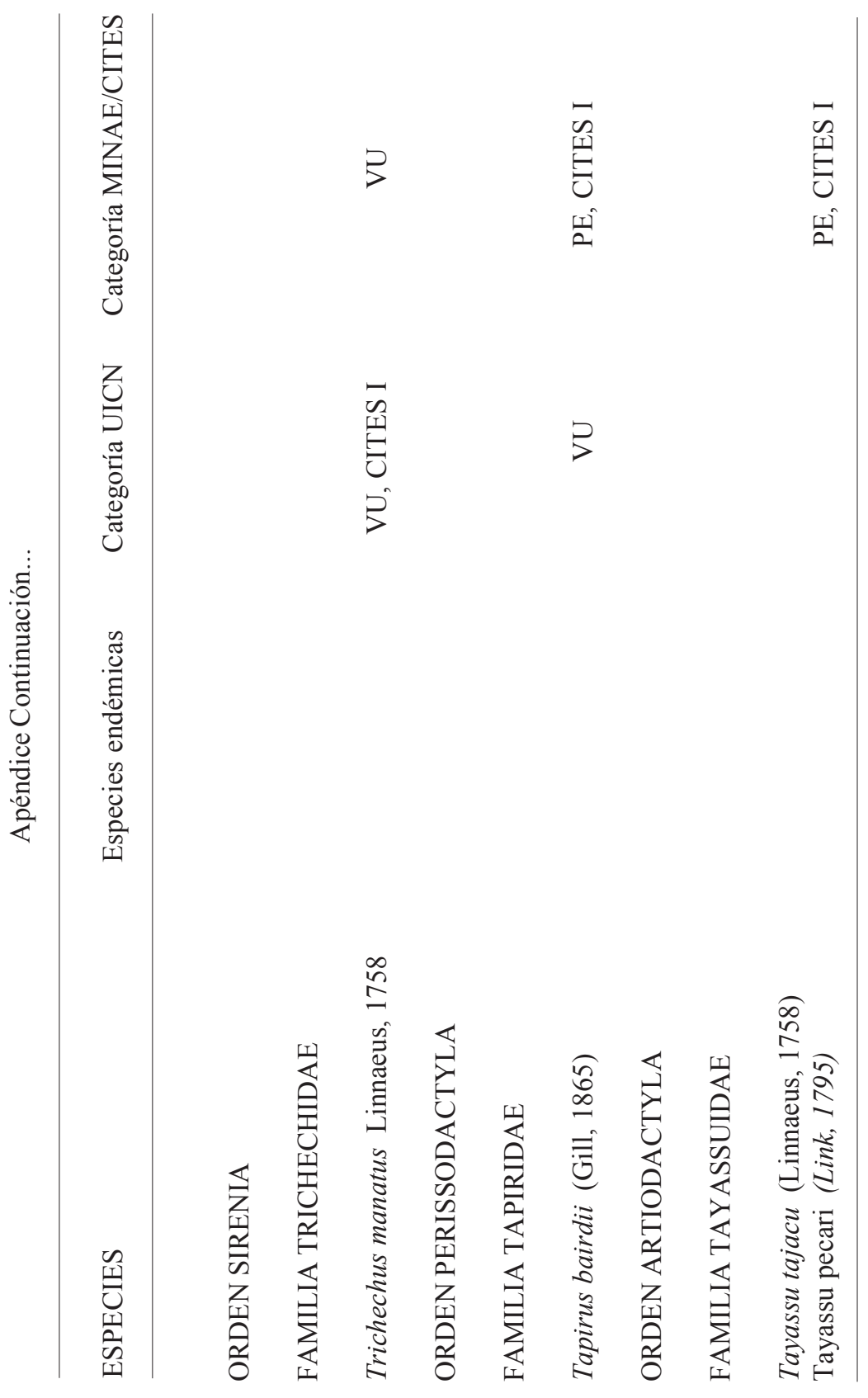



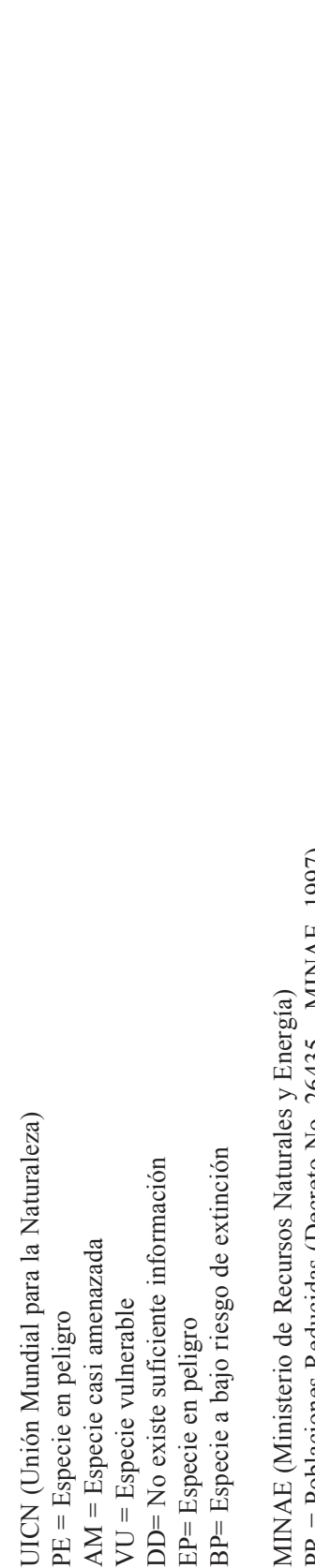